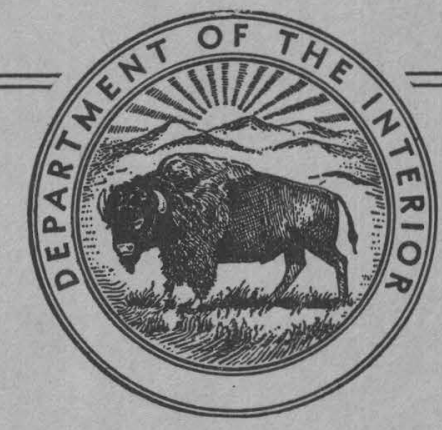

May 1951

\title{
INDEX OF SURFACE-WATER RECORDS
}

PART 8.-WESTERN GULF OF MEXICO BASINS

TO SEPTEMBER 30,1950

Prepared by Austin District 
UNITED STATES DEPARTMENT OF THE INTERIOR

Oscar L. Chapman, Secretary

GEOLOGICAL SURVEY

W. E. Wrather, Director

Washington, D. C.

Free on application to the Geological Survey, Washington 25, D. C. 


\section{INDEX OF SURFACE-WATER RECORDS}

\section{PART 8.-WESTERN GULF OF MEXICO BASINS TO EEPTHMBER 30,1950}

\section{ERTuntros}

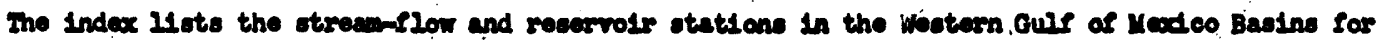
which reoords have been or are to be published for parlods pelor to sopterber 30, 1950. The stat10as are Listed in downstrean order. Iributary streane are indionted by indantion.

Many stations on canals and draine in the Blo Orando Beodn botwein Santa Fe and San Yaresal, N. I Iex., are not listed in this index. Heng stations on canale and ditches in the Peoce River Besin, dornatrean from Pecos, H. Yex., are not included in this index.

Station names are given in their most reoently publlahed focme. Parentheces around part of a otation name indicate that the Inclosed word or words wore used in an carlier publiched name of the otation or in a name under which records were published by acas ageney other than the Geolocteal surver.

The drainsge areas, in square mileo, are the latest 11 gurea published or otherulse arailable at this timo. Drainage areas that were obrlously inconsistent with other dralnage areas on the ame strean hare been conitted. Som drainage areas not published by the Geologieal surver are listed with ap appropelate foothote stating the published source of the flgure of dralnage area.

Under "portod of record" breake of 1038 than 12 -month perlod are sot ahown. A dach not followied immediately by a closing date show that the otation we in operation on soptember 30, 1950. The years given are calendar years. Pertode of records publiched by seasoles other then tho oeologieal survor are Iloted in parentheses only when thes contain more dotalled information or are for perslod not roported in publieations of the coological survey.

Records both of gege holght and of dischargo aro Iloted for otroamelow otatlons, and records of gage beight and of contents (or of ohange in contents) are lieted for otations on recervolre. Becords of gage

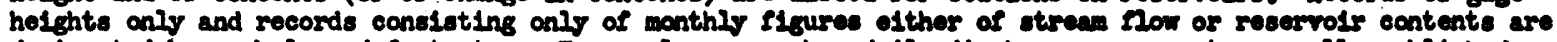
deslenated by oymbols and footnotes. For early years wen dally discharges were not generally publiehed by the Geological Survey, published dally gage helghte and a publiched rating table were conoldered to be equivalent to dails discharges.

An alphabetical index of otreama, canals, and resorrotre 1 fofven ca pages 18 to 21. 


\section{MGRUMETLAU RIVIRR BASIN}

Bayou Nezpique near Basile, La.................................... Cypress Creek near PIne Prairle, La.................................

Bayou des Cannes near Eunice, Ia..................................

Bayou Plaquemine Brule near Crowley, La..........................

Long Point Gully near Crowley, La..............................

\section{CAICASI HU HIVER BASIY}

Calcasieu River near Glenmora, Ia............................... Calcasieu River near Oberlin, La................................ Calcasieu River near Kinder, La................................. Whiskey Chitto Creek near Oberlin, La............................. Bundick Creek near Dry Creek, La............................... Beckwlth Creek near Dequincy, La................................ Hickory Branch at Kernan, La.................................

\section{SAB INE RIVRR BASII}

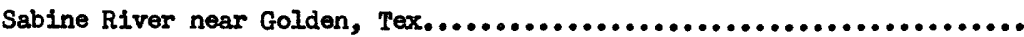
Sabine River near Mineola, Tex................................ Sabine River near Gladewater, Tex............................... Sabine River near Longview, Tex............................... Sabine River near Tatum, Tex................................... Sabine RIver at Logansport, La.................................

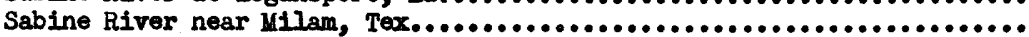
Sabine River near Sabinetown, Tex................................ Sabine River near Bon Wier, Tex.............................. Sabine River near Ruliff, Tex................................. Lake Fork Sabine River near Quitman, Tex. ....................... Big Sandy Creak near Big Sandy, Tex............................. Cherokee Bayou near Elderville, Tex. ............................. Lake Cherokee near Longview, Tex................................ Bayou San Miguel near Zwolle, La................................. Bayou Anacoco near Leesville, La.................................

\section{WECHES RIVRR BASIM}

Neches River near Reese, Tex.................................... Neches River near Neches, Tex................................... Neches River near Alto, Tex.................................... Neches River near Diboll, Tex.................................. Neches River near Rockland, Tex............................... Neches River at Evadale, Tex...................................

Mud Creek near Jacksonville, Tex..............................

Mud Creek at Ponta, Tex...................................

Lake Tyler near Whitehouse, Tex...............................

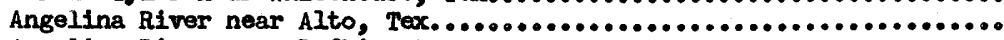
Angelina River near Lufkin, Tex................................. Angelina River at Horger, Tex....................................

Striker Creek near Summerfleld, Tex........................... Bowles Creak seepage Investigation........................... Attoyac Bayou near Chireno, Tex............................... Arenoso Creek near San Augustine, Tex......................... Aylsh Bayou at San Augustine, Tex...........................

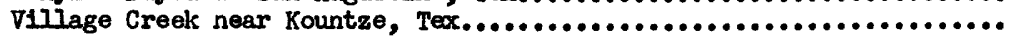

\section{TRIIITY RIVIR BASIN}

Bridgeport Resérvoir above Bridgeport, Tex........................ Trinity River, West Fork, at Bridgeport, Tex...................... Trinity River, West Fork, near Boyd, Tex............................. Eagle Mountain Reservoir above Fort Worth, Tex........................ Trinity River, West Fork, at Lake Worth Dam, above Fort Worth, Tex...... Trinity River, West Fork, at Fort Worth, Tex......................... Trinity River, West Fork, at Grand Prairle, Tex.......................
Drainage Area square miles)

$\begin{array}{ll}- & 1938- \\ - & 1948- \\ - & 1938- \\ - & 1942-47 \\ - & 1949-\end{array}$

$\begin{array}{cl}499 & 1943- \\ 753 & 1922-25 ; 1938- \\ 1,700 & 1922-25 ; 1938- \\ 510 & 1939- \\ 238 & 1939- \\ 148 & 1945- \\ 82.2 & 1945-\end{array}$

$\begin{array}{rl}1,199 & 1924-26 \\ 1,445 & 1939- \\ 2,846 & 1932- \\ 3,013 & 1904-06 ; 1923-32 . \\ 3,586 & 1939- \\ 4,858 & 1903-06 ; 1907-23 * * ; 1924- \\ 6,543 & 1939- \\ 6,754 & 1923-25 . \\ 8,323 & 1923-34 ; 1939- \\ 9,440 & 1924- \\ 586 & 1924-26 ; 1939- \\ 235 & 1939- \\ 116 & 1939-49 . \\ - & 1949-* * \\ - & 1948- \\ - & 1948-\end{array}$

851

1,129

1,903

2,670

3,539

7,908

382

490

1,274

1,575

3,435

148

$-502$

76

$-837$

1924-27.

1939-

$1944-$

1923-25; 1939-

1903-23**; 1924-

1904-06; 1923-

1939-

1924-27.

1949-**

$1940-49 * * *$.

1923-34; 1939-

1928-

$1940-49$.

1942.

1924-25; $1939-$

1938-40.

$1924-25$.

1924-27; 1939-

1,114

1,147

1,739

1,974

2,069

2,627

3,070

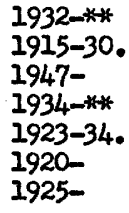

1932-***

1947-

1920 
Drainage Area

(square miles)

Trinity River at Dallas, Tex...................................

Trinity River near Rosser, Tex.................................. Trinity River near Oakwood, Tex.................................. Trinity River near Midway, Tex............................... Trinity River at Riverside, Tex............................... Trinity River at Romayor, Tex................................... Trinity River at Liberty, Tex..................................

Big Sandy Creek near Bridgeport, Tex..........................

Clear Fork Trinity River near Aledo, Tex..........................

Clear Fork Trinity River near Benbrook, Tex.......................

Clear Fork Trinity River at Fort ilorth, Tex......................

Marine Creek at Fort Worth, Tex.................................

Village Creek near handley, Tex..................................

Mountain Creek near Grand Prairie, Tex............................

Elm Fork Trinity River near Sanger, Tex..........................

Elm Fork Trinity River near Lewisville, Tex.........................

Elm Fork Trinity River near Denton, Tex........................

Lake Dallas near Lake Dallas, Tex...................................

Elm Fork Trinity River near Carrolltan, Tex.....................

Elm Fork Trinity River near Dallas, Tex........................... Isle du Bois Creek near Pilot Point, Tex......................... Clear Creek near Sanger, Tex................................ Denton Creek near Justin, Tex................................. Denton Creek near Roanoke, Tex............................... Denton Creek near Grapevine, Tex............................ East Fork Trinity River at McKinney, Tex..........................

East Fork Trinity River near Lavon, Tex..........................

East Fork Trinity River near Rockwall, Tex......................

East Fork Trinity River near Crandall, Tex....................... Sister Grove Creek near Princeton, Tex........................

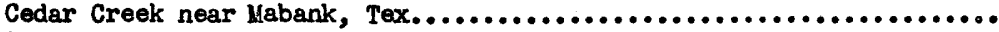

Chambers Creek near Emhouse, Tex...............................

Chambers Creek near Corsicana, Tex..............................

Richland Creek near Richland, Tex...........................

\section{SAN JACINTO RIVIR BASIN}

San Jacinto River, ilest Fork (San Jacinto River), near Conroe, Tex...... San Jacinto River, West Fork (San Jacinto River), near Humble, Tex....... San Jacinto River near Huffman, Tex............................. Spring Creek near Spring, Tex................................ Cypress Creek near Westfield, Tex...........................

East Fork San Jacinto River near Cleveland, Tex.................... Peach Creek at Splendora, Tex..............................

Caney Creek near Splendora, Tex.............................

Barker Reservoir near Clodine, Tex...............................

Barker Reservoir near Addicks, Tex............................ Buffalo Bayou near Addicks, Tex...................................

Buffalo Bayou at Houston, Tex.................................. Addicks Reservoir near Addicks, Tex............................. Whiteoak Bayou at Houston, Tex................................. Brays Bayou at Houston, Tex..................................

CLEAR CRYGK BASIM

Clear Creak near Pearland, Tex.....................................

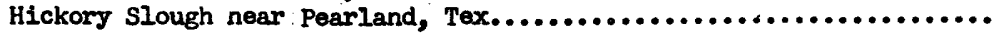

\section{CHOCOLATR BAYOU BASIX}

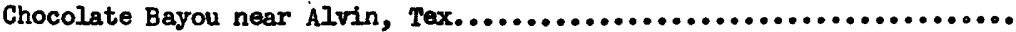

\section{BASTROP BAYOU BASIN}

\section{6,120}

8,162

12,912

14,484

15,619

17,192

17,539

332

246

435

526

16.8

126

273

379

1,671

1,076

1,165

2,534

2,577

261

296

409

621

704

188

779

840

1,257

115

734

818

977

737

832

1,811

2,791

400

262

330

120

104

105

150

310

362

129

92

100

\section{Period of Record}

1898-99*; 1903-06;

1907-20k*; 1920-

1924; 1924-25*; $1938-$

1905-23**; 1923-

1939-

1903-06; 1923-

1924-

1938-40*; 1940-***

1936-

1947 -

$1947-$

1924-

1950-

1925-30.

1925-33.

1949-

1949-

1923-27.

1928-

1923-

1920-28.

1949-

1949-

1949-

1923-27; $1939-$

1947-

1949-

1949-

1923-

$1949-$

1949-

1938-

$1924-25$.

1939-

1924-25*; 1939-

$1924-27 ; 1939-$
$1928-$
$1936-$
$1939-$
$1944-$
$1939-$
$1943-$
$1944-$
$1945-$
$1945-* *-$
$1945-$
$1936-$
$1948 \ldots *$
$1936-$
$1936-$

1944***; 1946***; 1947-*** 1944***; 1946***; 1947-49*k*.

1944***; 1946 **** 


\section{OYSTER CRTMX BASIN}

Oyster Creek near Angleton, Tex.

\section{Drainage Area (square miles)}

\section{BRAZOS RIVER BASIN}

Brazos River, Double Mountain Fork, at Lubbock, Tex................... Brazos River, Double Mountain Fork, near Rotan, Tex................... Brazos River, Double Mountain Fork, near Aspermont, Tex................ a

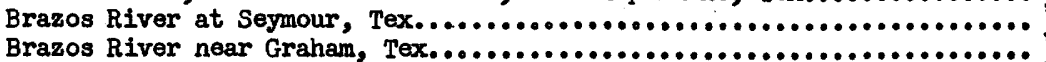

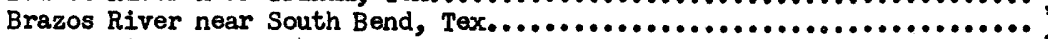
Possum Kingdon Reservoir near Graford, Tex........................

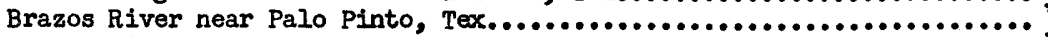

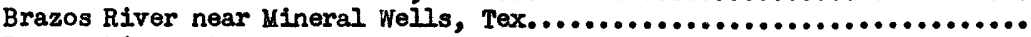
Brazos River at Brazos, Tex.................................... Brazos River near Glen Rose, Tex.............................. Brazos River near Whitney, Tex................................ Brazos River at Waco, Tex......................................

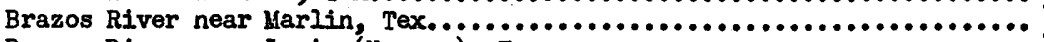
Brazos River near Lewis (Hearne), Tex............................

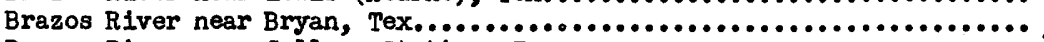

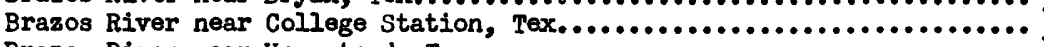

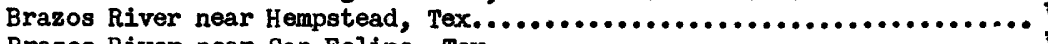

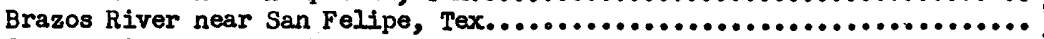

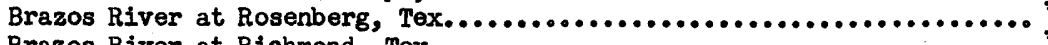

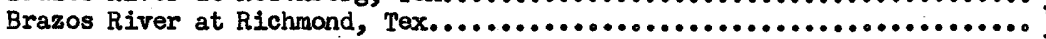

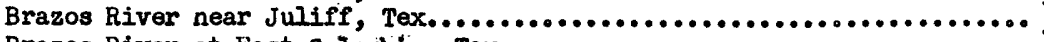

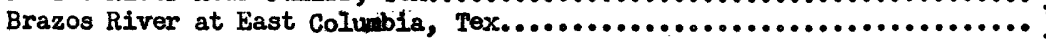

Salt Fork Brazos River near Peacock, Tex.......................... Salt Fork Brazos River near Aspermont, Tex....................... White River at Plainview, Tex................................ Clear Fork Brazos River at Nugent, Tex.......................... Clear Fork Brazos River at Fort Griffin, Tex...................... Clear Fork Brazos River at Crystal Falls, Tex......................

Clear Fork Brazos River near Crystal Falís, Tex.....................

Clear Fork Brazos River near Eliasville, Tex......................

Paint Creek near Haskell, Tex.............................

Fort Phantom Hill Reservoir near Nugent, Tex....................

Palo Pinto Creek near Santo, Tex..............................

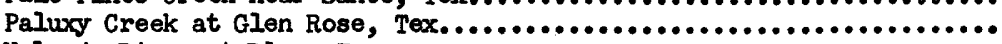
Nolands River at Blum, Tex................................... Aquilla Creek near Aquilla, Tex............................... North Bosque River near clifton, Tex........................... South Bosque River near Speegleville, Tex...................... Deer Creek at Chilton, Tex................................... Leon River near Eastland, Tex............................. Leon River near Hasse, Tex................................... Leon River near Hamiltion, Tex................................. Leon River near Belton, Tex.................................. Iittle River near Little River, Tex........................... Little River at Cameron, Tex.............................. Cowhouse Creek near Killeen, Tex.............................. Lampasas River at Youngsport, Tex............................ Sulphur Creek seepage investigations........................ San Gabriel River at Georgetown, Tex......................... San Gabriel River at Circleville, Tex........................

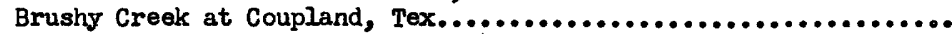
Big Elm Creek near Temple, Tex.............................

Big EIm Creek near Buckholtz, Tex............................ North Elm Creek near Ben Arnold, Tex.

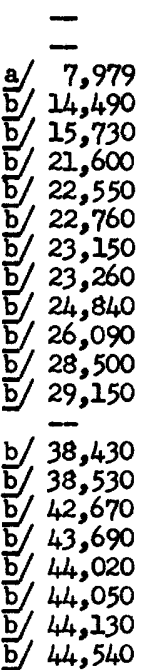

c) 4,8 $\overline{4}, 834$ $\overline{2}, 220$ 3,974

4,323

5,658

5,740

$-478$ 507

399

275

309

974

388

81.8

$-$

1,276

1,895

3,547

5,253

7,034

650

1,242

415

602

198

68.5

166

30.3
Period of Record

1944-

1939-49.

1949-

1923-34; 1939-

1923-

1915-20.

1938-

1941- $x$ *

1933-

$1924-33$.

$1914-20$.

1923-

1938-

1898-1911; 1912-14**; 1914-

1938-

$1898-99 \%$.

1925-

$1918-25$.

1938-

1938-45; 1945-*

1922-31.

1903-06; 1931-

1949-

1938-39*; 1939-40***;

1940-41*; 1942-k***

1949-

1923-25; 1939-

1939-49.

1924-

1923-

1921-29.

1928-

1915-20; 1923-25.

1949-

1940-k*k

1924-25.

$1923-25$; $1947-$

1924-25; 1947-

1924-25; 1938-

1923-

$1924-30$.

1934-36.

1950-

1939-

1925-31.

1923-

1923-29.

1916-

1924-25; $1939-42$.

1924-

1942.

1924-25; $1934-$

1924-34.

1924-26.

1934-36.

1934-36.

1934-36.

* Gage heights or gage heights and discharge measurements only.

* *nly monthly figures of discharge or contents.

*tis: Fragmentary.

a/ 6,470 square miles probably noncontributing.

b/ 9,240 square miles probably noncontributing.

c/ 2,770 square miles probably noncontributing. 
Brazos River tributaries-Contimed

Yegua Creek near Somerville, Tex.

Navasota River near Easterly, Tex. American Canal Co.'s canal (Brazos Valïey Irrigation Co.'s Canal)

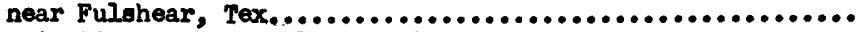
RIchmond Irrigation Co. is canal near Rlchmond, Tex..................

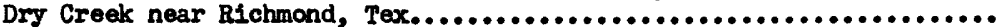
Big Creek near Needville, Tex.................................. Big Creek near Guy, Tex.......................................... Fairchild Creek near Needvilie, Tex...........................
Drainage Area

(square miles)

\begin{tabular}{|c|c|}
\hline $\begin{array}{l}990 \\
949\end{array}$ & $\begin{array}{l}1924- \\
1924-\end{array}$ \\
\hline & $\begin{array}{l}1931- \\
1931-\end{array}$ \\
\hline $\begin{array}{c}10.3 \\
37.6 \\
112 \\
24.9\end{array}$ & $\begin{array}{l}1947-50 \\
1947-50 \\
1947-50 \\
1947-\end{array}$ \\
\hline
\end{tabular}

\section{COLORADO RIVHR BASIY}

Colorado River near Ira, Tex.................................. d 3,617 Colorado River at Colorado City (Colorado), Tex..................... d/ 4,082 Colorado River at Robert Lee, Tex................................ Colorado River near Robert Le日, Tex.............................. e/ 15,860 Colorado River near Bronte, Tex................................. e/ 15,940 Colorado River at Ballinger, Tex............................. e/ 16,840 Colorado River at Hinchell, Tex................................... I / 24,580 Colorado River near Milburn, Tex................................ Colorado River near San Saba (Chadwick), Tex........................ Colorado River near Tow, Tex....................................

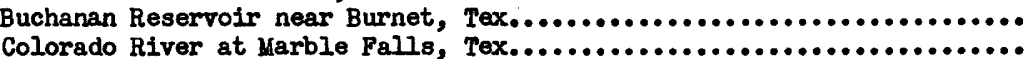
Lake Travis (Marshall Ford Reservoir) near Austin, Tex................. Colorado River at dam above Austin, Tex........................... Colorado River at Austin, Tex.................................... Evaporation at (near) Austin, Tex...............................

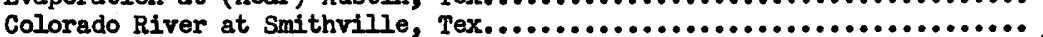
Colorado River at La Grange, Tex.............................. Colorado River at Columbus, Tex.................................

Colorado River near Eagle Lake, Tex.............................. Colorado River at Wharton, Tex.................................... Colorado River near Bay City, Tex................................. Colorado River seepage investigations.......................... Bull Creek near Ira, Tex.................................... Bluff Creek near Ira, Tex...................................... Deep Creak near Snyder, Tex.................................... Morgan Creek near Colorado City, Tex............................. Lake Colorado City near Colorado City, Tex......................... Champlin Creek near Colorado City, Tex............................. Oak Creek near Blackwell, Tex.................................. Elm Creek at Ballinger, Tex..................................... South Concho River (head of Concho River) at Christoval, Tex..........

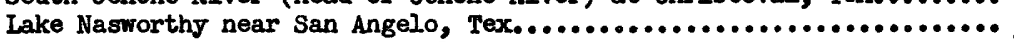

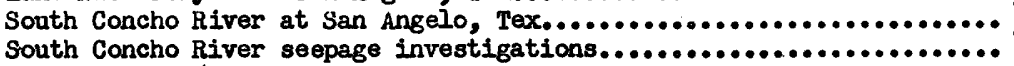
Concho Rivar near San Angelo, Tex............................. g Concho River near Paint Rock, Tex................................. Concho River seepage investigations............................. South Concho Irrigation Co.'s canal at Christoval, Tex..............

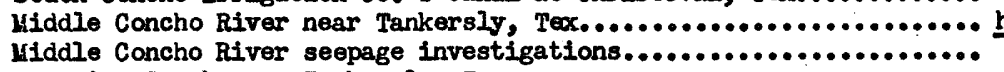
Spring Creek near Tankersly, Tex............................. Spring Creek seepage investigations............................. Dove Creek near Knickerbocker, Tex........................... Dove Creek seepage investigations........................... Dove Creek Springs near Knickerbocker, Tex.................. r) 24,600
1947-

1923-25; $1946-$

1939-

1923-27. 1915-18; 1918-20*. 1907-15**; 19151939-

1923-34.

1915-22; $1930-$

1923-34.

$1937-* *$

1916-26.

1940-***

1895-98.

1898-

1911, 1916-39.

1930-

1938-

1903*; 1904-11; 1916-30; 1939-

1930-39.

1916-25; 1938-

1948-

1918; 1925.

1947-

1947-

1923-25.

1947-49.

1950-**

1947-

1949-

1932-

1930-

1930-**

1931-

1918 ; 1925.

1915-

1915-

$1918 ; 1925$.

1939-

1930-

1918 ; 1925.

1930-

1918 ; 1925.

$1944-49$.

1925.

1944- At

* Gage heights or gage heights and discharge measurements only.

** Only monthly figures of discharge or contents.

Hf Discharge measurements only at regular intervals.

d) 2,590 square miles probably noncontributing.

e/11,600 square miles probably noncontributing.

f/11,900 square miles probably noncontributing.

g/ 275 square miles probably noncontributing.

h/ 152 square miles probably noncontributing. 
Colorado River tributaries-Contimued

Concho River tributaries-Continued

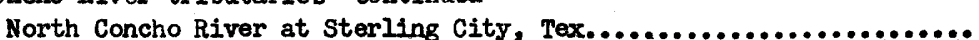

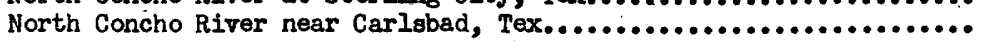

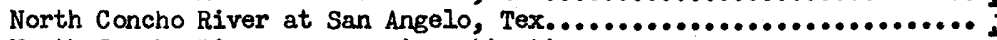

North Concho River seepage investigations.........................

Brownwood Reservoir near Brownwood, Tex...........................

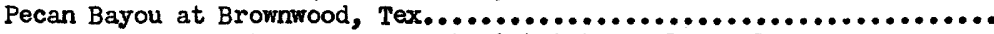
Brown County Water Improvement District No. I Canal near

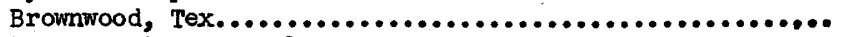
Hords Creek Reservoir near Valera, Tex......................... Hords Creek near Valera, Tex................................ Hords Creek at Coleman, Tex................................. San Saba River at Menard, Tex...............................

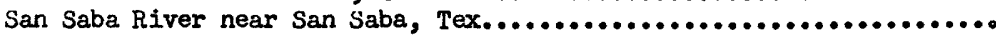
San Saba River at San Saba, Tex................................

San Saba River seepage investigations............................. Noyes Canal at Menard, Tex.....................................

Brady Creek at Brady, Tex.................................. North Llano River near Junction, Tex.............................. North Llano River seepage investigation........................... Llano River near Junction, Tex................................ Llano River near Castell, Tex.................................

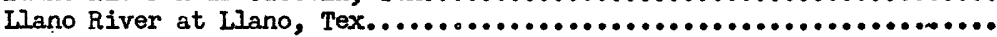
Llano River seepage investigations.............................

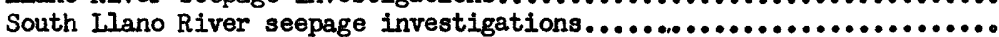
Johnson Fork Llano River seepage investigations.................... Pedernales River at Stonewall, Tex............................ Pedernales River near Johnson City, Tex..........................

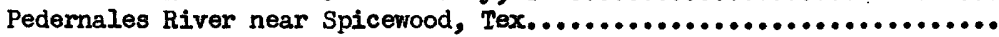
Barton Creek. at Austin, Tex................................. Barton Springs at Austin, Tex............................. Ifittle Walnut Creek near Austin, Tex........................... Onion Creek near Del Valle, Tex............................... Dry Creek at Buescher Lake, near Smithville, Tex....................

\section{IATACA RIVIR BASIV}

Lavaca River at Hallettsville, Tex.............................. Lavaca River near Edna, Tex............................... Lavaca River seepage investigation............................... Navidad River near Ganado, Tex.................................

\section{GUADALUPE RI VHR BASIN}

Guadalupe River at Hunt, Tex................................. Guadalupe River near Comfort, Tex................................ Guadalupe River at Comfort, Tex.................................

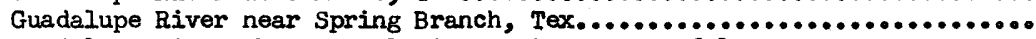
Guadalupe River above Comal River, at New Braunfels, Tex............... Guadalupe River at New Braunfels, Tex............................. Guadalupe River near Gonzales, Tex.............................. Guadalupe River near Cuero, Tex............................... Guadalupe River below Cuero, Tex.............................. Guadalupe River at Victoria, Tex............................. Guadalupe River seepage investigation.............................. Johnson Creek near Ingram, Tex................................ Hueco Springs near New Braunfels, Tex............................ Comal River at New Braunfels, Tex.......................... k

San Marcos Springs at San Marcos, Tex............................. San Marcos River at San Marcos, Tex............................ k

San Marcos River at Luling, Tex.................................

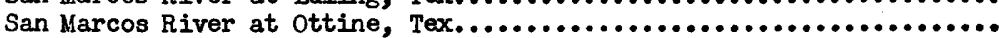
Blanco River at Wimberley, Tex..............................
Drainage Area

(square miles)

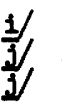

690

1,529

1,795

1.535

1,614

$-48$

53

107

1,151

3,038

3,046

$-$

575

914

-

1,762

3,514

4,000

-

647

947

1,294

125

$-$

12

337

1.48

\section{Period of Record}

1939-

$1924-$

1915-31; 1947-

1918 ; 1925.

1933-41**; 1944-**

1917-18; 1923-

1950-

$1948-* * *$

1947-

1940-

1915-

1904-06; 1915-30.

$1930-$

1918; 1933; 1940.

1924-

1939-

1915-

1925.

1915-

1923-39.

1939-

1918 ; 1925.

1918; 1925.

1925.

$1924,-34$.

1939-

1923-39.

1917-18.

1917- 44

1924-26.

$1924-30$.

1939-

$\begin{array}{rr}101 & 1939- \\ 887 & 1938- \\ -1,116 & 1948- \\ -1939-\end{array}$

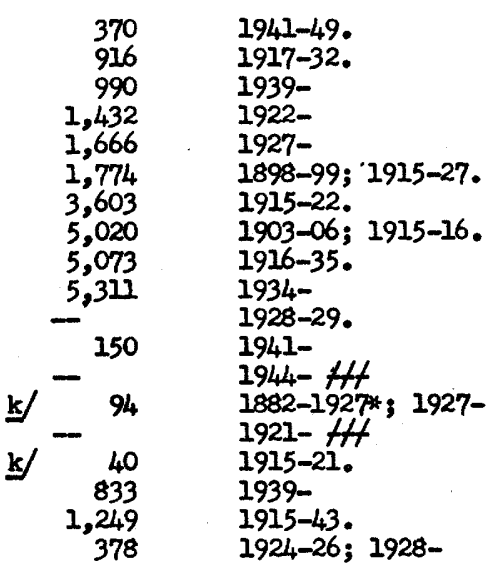

* Gage heights or gage heights and discharge measurements only. *** Only monthly figures of discharge or contents.

HA Discharge measurements only at regular intervals.

if 75 square miles probably noncontributing.

$\bar{j} / 123$ square miles probably noncontributing.

k/ Normal flow of river comes from springs; drainage area of stream not applicable. 
Guadalupe River tributaries-Contimed

San Marcos River tributaries-Continued

Blanco River seepage Investigation...............................

Plum Creek near Lockhart, Tex...............................

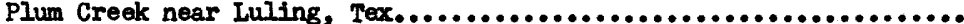

Peach Creek near Dilworth, Tex................................ Sandies Creek near Westhoff, Tex.................................. Coleto Creek near Schroeder, Tex............................... Coleto Creek near Victoria, Tex.................................. San Antonio River at San Antonio, Tex........................... k

San Antonio River at Calaveras, Tex............................. San Antonio River near Falls City, Tex............................ San Antonio River at Goliad, Tex...............................

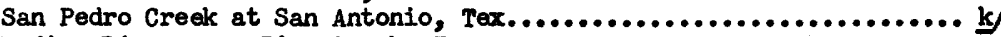
Medina River near Plpe Creek, Tex............................ Medina Reservoir (Lake) near San Antonio, Tex..................... Medina River near Riomedina, Tex..............................

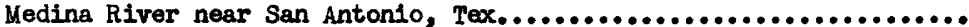
Medina River seepage past station near Riomedina, Tex............... Medina River seepage investigations.............................. Medina Canal near Riomedina, Tex............................ Cibolo Creek near Bulverde, Tex................................. Cibolo Creek above Bracken, Tex................................

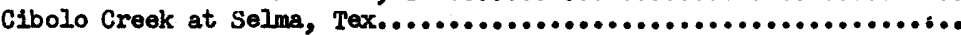
Cibolo Creek at Sutherland Springs, Tex....................... Cibolo Creek near Falls City, Tex.............................. Cibolo Creak seepage investigation..............................

\section{MISSIOI RIVIR BASIY}

Mission River at Refugio, Tex..................................

\section{WUDCES RIVIHR BASIM}

Nueces River at Laguna, Tex......................................... Nueces River near Uvalde, Tex.................................. Nueces River below Uvalde, Tex................................ Nueces River near Cinonia, Tex................................ Nueces River near Asherton, Tex............................... Nueces River near Cotulla, Tex.................................. Nueces River at Cotulla, Tex.................................... Nueces River near Tilden, Tex..................................

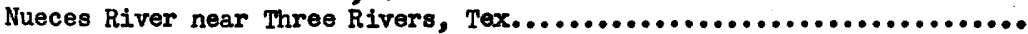
Lake Corpus Christi near Mathis, Tex.............................. Nueces River near Mathis, Tex................................... Nueces River at Calallen, Tex................................ Nueces River seepage investigations..............................

West Nueces Rirer near Brackettville, Tex........................ West Nueces River seepage investigation............................ Frio River at Concan, Tex.................................. Frio River near Frio Town, Tex................................

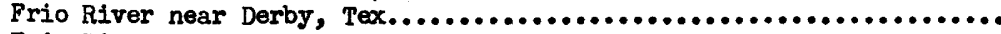
Frio River at Fowlerton, Tex.................................... Frio River at Calliham, Tex.................................. Frio River at Three Rivers, Tex................................. Frio River seepage investigations............................. Dry Frio River seepage investigations........................... Sabinal River near Sabinal, Tex.............................. Sabinal River seepage investigations.......................... Leona River spring flow near Uvalde, Tex........................ Leona River near Divot, Tex.................................. Leona RIver seepage investigations.............................

Frio Lake Outlet near Fowlerton, Tex............................ Atascosa River at initsett, Tex...............................
Drainage Area square miles)

-184
356
445
493
365
514
42
1,786
2,071
3,918
2.64
412
587
606
1,225
$-\quad$
$-\quad$
$-\quad 198$
251
280
665
831
-

643

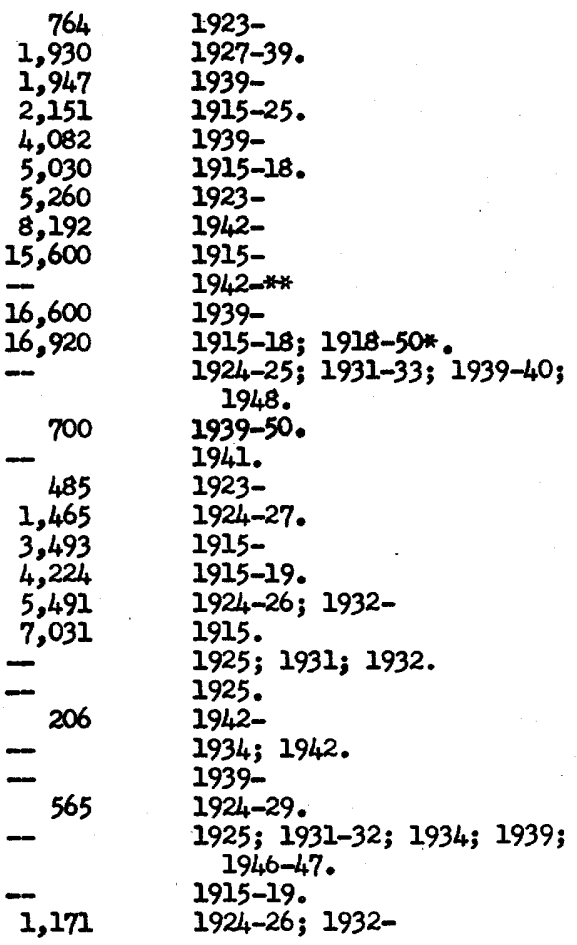

\section{Period of Record}

1924.

1925-30.

1930

1930-33.

1930-34.

1930-33.

1939-

1915-29; 1939-

1918-25.

1925-

1916-29.

1922-34.

1913-**

1922-34.

1939-

1922-34.

1925; 1929-30.

1922-34.

$1946-$

1946-

1946-

1924-29.

$1930-$

1949.

1939-
1924-29; $1939-$

* Gage heights or gage heights and discharge measurenents only.

* Only monthly figures of discharge or contents.

k/ Normal flow ot river comes from springs; drainage area of stream not applicable. 


\section{RIO GRANDI BASI}

Rio Grande at Thirtymlle Bridge near Creede, Colo.................... Rio Grande at Wason, below Creede, Colo......................... Rio Grande near Del Norte, Colo.................................

Rio Grande near Monte Vista, Colo............................... Rio Grande at Alamosa, Colo................................... Rio Grande above mouth of Trinchera Creek, near La Sauses, Colo........ Rio Grande near Lobatos, Colo...................................

Rio Grande near Cerro, N. Hex................................... Rio Grande below (at) Taos Junction Bridge near Taos, N. Mex............ Rio Grande at Embudo, N. Mex.....................................

Rio Grande at Otowl Bridge $n /$ near San Ildefonso, N. Mex................

Rio Grande at (near) Cochiti, N. Mex............................ Rio Grande at San Felipe, N. Mex.............................. Rio Grande near Bernalilio, N. Mex............................ Rio Grande at Albuquerque, N. Hex. ............................ Rio Grande near (at) Isleta, N. Mex.............................

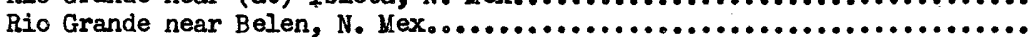
Rio Grande near Bernardo, N. Mex............................. Rio Grande at San Acacia, N. Mex............................... Rio Grande at (near) San Marcial, N. Hex............................

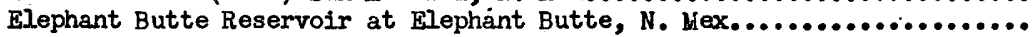
Rio Grande below Elephant Butte Dam, N. Mex........................ Caballo Reservoir near Arrey, $N$. Mex............................. Rio Grande below Caballo Dam, N. Mex,.......................... Rio Grande at Percha Dam, near Arrey, N. Mex...................... Rio Grande at Leasburg Dam, near Fort Seldon, N. Kex.................

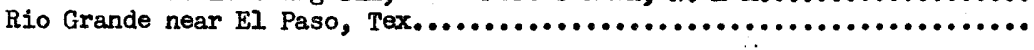

Rio Grande below American Dam, Tex........................... Rio Grande at Cd. Juarez, Ch1hnahwa, Mex........................... Rio Grande at Island tation near ti Paso, Tex................... of

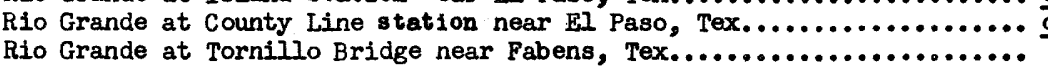

Rio Grande near Fort Hancock, Tex............................ Rio Grande below old Fort Quitman near Finlay, Tex................... of 31,990

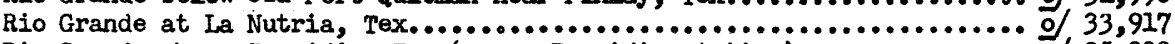
Rio Grande above Presidio, Tex. ( upper Presidio station).............. of 35,000

Rio Grande below Presidio, Tex.( low er Presidio station)............ of 59,767

Rio Grande at Johnson Ranch, Tex.......................... of 66,451

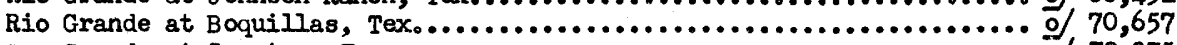
Rio Grande at Iangtry, Tax................................. of 79,375

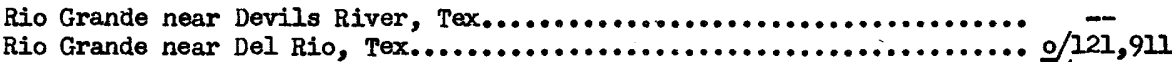

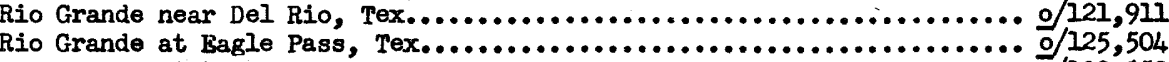
Rio Grande at Laredo, Tex.................................... $0 / 130,859$ Rio Grande at Zapata, Tex................................... $0 / 154 ; 426$ Rio Grande at Roma, Tex.................................. $0 / 157,448$

Rio Grande at Rio Grande City, Tex.............................. o/170,986 Rio Grande at Hidalgo, Tex.................................. $0 / 171,831$ Rio Grande at Las Palmas, Tam., kex............................... Rio Grande at Buenos Aires, Tam., Mex.............................

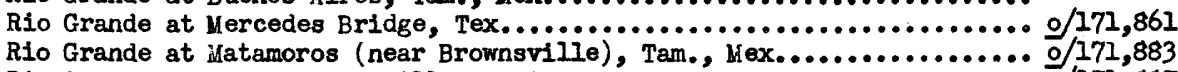
Rio Grande at Lower Brownsville Station, Tex..................... o /171,887 Rio Grande seepage investigations. 16,100 17,750 19,230 27,700
Period of Record

1909-13; (1913-33)m/; 1933$1907-13 ;(1913-33) \mathrm{m} / ; 1933-$ 1889-1906; 1908-13;

$(1913-33) \mathrm{m} /$; $1933-$

(1926-33)m ; 1933-

1912-13; (1913-33)m/; $1933-$ 1936-

1899-1913; (1913-33)m/; 1933-

1948-

(1925; $1926-30) \mathrm{m} / 1930-$

1889-1903; 1912-16;

(1916-30)m/; $1930-$

1895-1905; 1909-14;

$(1915-30) \mathrm{m} /$; $1930-$

(1925**; 1926-30)m/; 1930

$(1926-30) \mathrm{m} /$; $1930=$

1941-

$1942-$

1936-38.

1942-

1936-39; 1941-

(1925**; 1926-27*)m/; 1936-

1895-

(1924-39**)o/; 1940-

1916-

2938-

1938-

(1926-31)m/.

$(1926-31) \mathrm{m} /$

1889-93; (1894)o/;

1895-1931; (1932-)o/

(1938-)의

(1938-) 의

(1938-)의

(1938-)

(1924-27)야; 1927-31;

$(1931-37)$ o

1900-03.

1923-31; (1931-)o/

(1935-4I)o\%.

1900-14; 1919-20; 1923-26; (1926-)o/

1900-15; 1919-20; 1923-26; (1924-)o

(1936-) 0

1928-31; (1931-36) \%

1900-14; 1919-20; 1924-31;

(1931-)o/

1900-15; 1919-20.

1923-31; (1931-)o/

1900-16; 1923-31; (1931-)의

1900-14; 1922-26; (1923-)의

(1932-) o/

1900-14; 1922-25; 1929-31;

(1924-29)의; (1931-)o/

(1924-)의

1928-31.

(1945-49)\%

(1943-44)\%

$(1935-41) \%$

1900-14; I922-26; (1926-)o/

(1934-)o/

$1925-26 ; 1928$. 
Rio Grande tributaries

Clear Creek below Continental Reservoir, Colo.........................

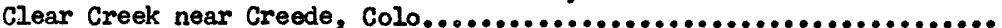

Goose Creek near ilagonwheel Gap, Colo.............................. South Fork Rio Grande at South Fork, Colo.......................... Pinos Creek near Del Norte, Colo.................................... San Francisco Creek near Del Norte, Colo............................... Empire Canal below Bowen Drain erossing, near Alamoas, Colo........... Bowren drain below Empire Canal crossing, near Alamosa, Colo........... Rock Creek near Honte Vista, Colo...................................

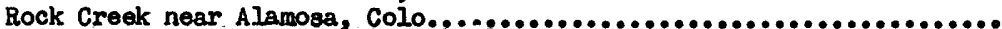
Closed basin in San Irais Valley, Colo.:

San Luis Creak at Villa Grove, Colo..............................

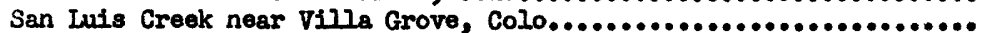
Kerber Creek at Ashley Ranch, near Villa Grove, Colo................ Kerber Creek near Villa Grove, Colo.............................. Kerber Creek below (near) Villa Grove, Colo..................... Saguache Creek near. Saguiche, Colo................................ North Crestone Creek near Crestone, Colo............................ South Crestone Creek near Crestone, Colo.......................... Willow Creek near Crestone, Colo................................. Spanish Creek near Crestone, Colo............................... Cottonwood Creek near Crestone, Colo.............................. Deadman Creek near Crestone, Colo............................... Carnero Creek near Ia Garita, Colo................................ La Garita Creek near La Garita, Colo.............................. North lateral Farmers Union ditch near Hooper, Colo.................. Farmers Union drain near Hooper, Colo............................ Arena (Sand) Creek near Crestone, .. Colo............................ BIg Spring Creek near Hooper, Colo................................ Little Spring Creek near Hooper, Colo............................ Prairie ditch near Mosca, Colo................................... North Zapato Creek near Blanca, Colo.............................. Seepage and waste from Rio Grande into closed basin.................

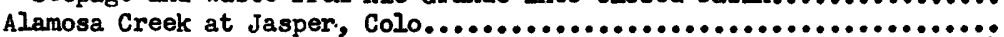
Alamosa Creek above Terrace Reservoir (near Monte Vista), Colo........

Alamosa Creek below Terrace Reservoir (near La Jara), Colo...........

Alamosa Creek near Capulin, Colo............................... La Jara Creek at Gallegos Ranch, near Capulin, Colo.................. La Jara Creek near Capulin, Colo................................

La Jara Creek below Empire Canal, near Sanford, Colo................. La Jara Creek at mouth, near Alamosa, Colo........................... La Jara drain near Sanford, Colo............................... Trinchera Creek above Turners Ranch, near Fort Garland, Colo.......... Trinchera Creak above Mountain Home Reservoir, near Fort Garland,

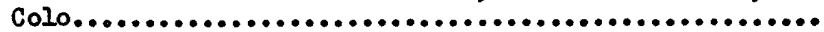
Trinchera Creek below Smith Reservoir near Blanca, Colo............. Trinchera Creek at mouth, near La Sauses, Colo....................... Sangre de Cristo Creek near Fort Garland, Colo....................... Sangre de Cristo Creek above Smith Reservoir, near Blanca, Colo.....

Ute Creek at upper station, near Fort Garland, Colo...............

Ute Creek near Fort Garland, Colo...............................

Conejos River at Platoro, Colo.................................. Conejos River at Counseliors Cabin, near Mogote, Colo..................

Conejos River near Mogote, Colo.....................................

Conejos River near La Sauses, Colo................................. San Antonio River near Ortiz, Colo............................... San Antonio River at ortiz, Colo...............................

San Antonio River at mouth, near Manassa, Colo....................

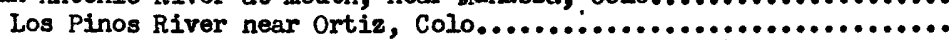
Culebra River near Chama, Colo.................................
Drainage Area square miles)

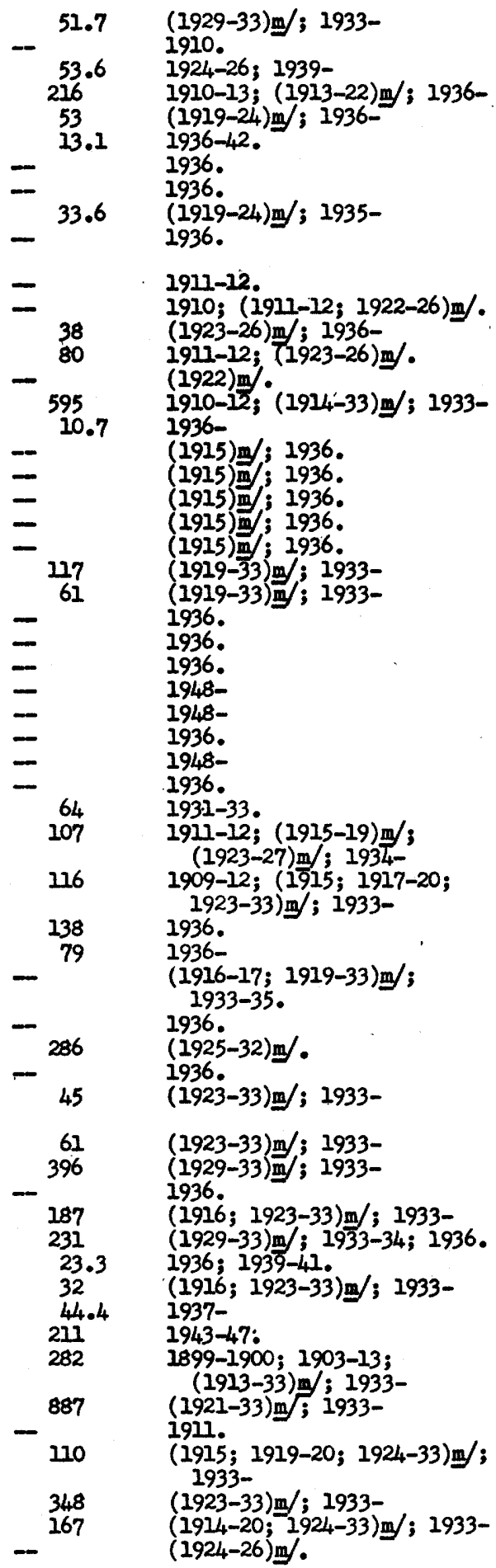


Rio Grande tributaries-Contimued

Culebra Creek at San Luis, Colo....................................

Culebra Creek below San Luis, Colo................................ Culebra Creek at mouth, near San Acacio, Colo.......................... Costilla Creek above reservoir near Costilla, N. Ilex................. Costilla Creek below reservolr near Costilla, N. Uex................ Costilla Creek near Amalia, No Hex.................................. Costilla Creek near Costilia, N. Mex............................ Costilla Creek at Garcia, Colo................................. Costilla Creek near Jaroso, Colo (near mouth, N. Ulex.)...............

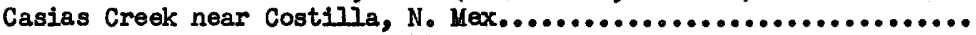
Santistevan Creek near Costilla, N. Mex........................ Ute Creek near Amalia, No Mex.................................. Acequia Hadre at Costilla, N. Mex...........................

Mesa ditch near Garcia, Colo................................. Middle ditch at Garcia, Colo................................ Cordillera ditch at Garcia, Colo...............................

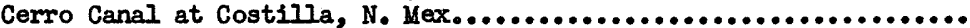
Cerro Canal near Jaroso, Colo..................................

New Mexico Branch Cerro Canal near Jaroso, Colo................... Alire ditch at Garcia, Colo.................................... Eastdale No. I Intake Canal near Jaroso, Colo........................ Iatir Creek near Cerro, N. Mex................................. Red River (Rio Colorado) near Red River, N. Mex..................... Red River (Rio Colorado) above Questa, N. Yex...................... Red River (Rio Colorado) near Questa, N. Mex......................

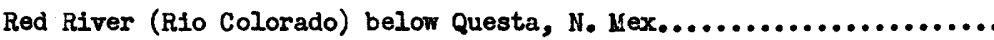

Cabresto Creek near Questa, N. Mex............................

Llano ditch near Questa, No Mex............................. Rio Hondo near Valdez, N. Mex................................ Rio Hondo at Valdez, N. Mex..................................

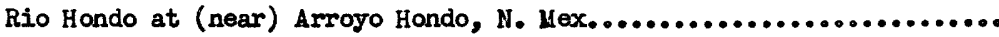

Rio Pueblo de Taos near Taos, N. Mex.............................. Rio Pueblo de Taos at Taos, N. Yex................................ Rio (Pueblo de) Taos at Los Cordovas, No Hex........................

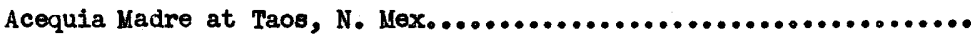
North channel of Rio Pueblo de Taos at Taos, N. Mex................. Rio Lucero near (Taos) Arroyo Seco, N. Mex......................... Rio Iucero below diversions near Arroyo Seco, N. Mex................ Tenorio ditch near Arroyo Seco, N. Mex.......................... Rio Lucero diversions near Arroyo Seco, $\mathrm{N}$. Mex................... Indian ditch near Arroyo Seco, $N$. Mex.......................... Seco ditch near Arroyo Seco, N. Mex............................ Juan Manual ditch near Arroyo Seco, N. Mex........................

Prado ditch near Arroyo Seco, N. Mex...........................

Rio Fernando de Taos near Taos, N. Hex..........................

Rio Ranchos de Taos near Ranchos de Taos, N. Mex...................

Carson Reservoir near (Stong) Carson, N. Mex....................... Embudo Creek at Dixon, N. Mex.................................

Pueblo Creek near Penasco, N. Mex..............................

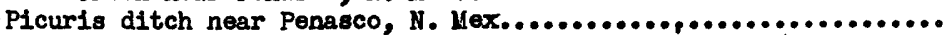
Alcalde ditch (at Alcalde) near Chamita, N. Yex......................

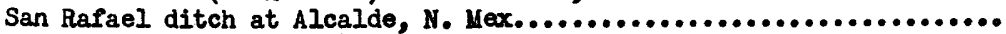
Acequia Madre at Alcalde, N. Mex...............................

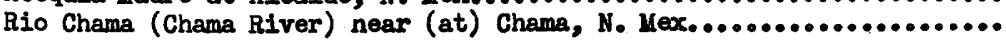

Drainage Area

(square miles)
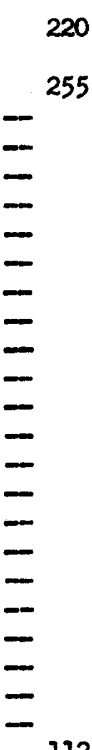

112

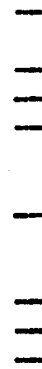

三

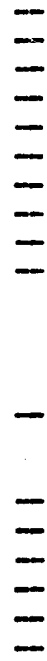

64

149
Period of Record

(1909) $\mathrm{m} /$; 1910-11;

$(1927-33) \mathrm{m} /$; $1933-$

1938-

1936.

1937-

1937-

1948-

1936-

$1944-$

1912-13**; 1936; 1948-

1937-

2937-

1948-

$1944-* *$

1944-***

$1944-* *$

$1944-* * *$

1944-***

$1944-* * *$

$1944-* *$

$1944-* * *$

$1944-* *$

$1937-$

1940

1910-11*。

1912-15; (1915-24; 1924-25**;

$1926-30) \mathrm{m} /$; 1930

1910-15; (1915-24;

1924-25**; $1926-28) \mathrm{m} /$.

1943-

$1943-* *$

1934-

$38 \quad$ (1916-24; 1924-25**; $1926-30) \mathrm{m} /$; $1930-34$.

1910-12*; I912-15; (1915-24;

1924-25**; 1926-28)m/; 1932-

1910*; 1911-16; 1940-

$1936-41$.

1910-15; (1915-24;

1924-25**; 1926-30)m/;

1930-

1940-41**。

1936-4l.

1910\%; 1911-16; 1933-

1934-42.

1935-39; 1940-**

1932-33.

1934-39; 1940 -***

1934-39; 1940-**

1935-39; 1940-***

1934-39; 1940-***

1910*; 1912-15; (1915-24

$1924-25 * * ; 1926-28) \mathrm{m} /$.

(1920-24; 1924-25**;

1926) $\mathrm{m}$.

1940

$305 \quad(1923-24 ; 1924-25 * *$;

$1926-30) \mathrm{m} /$; $1930-$

$1936-41$.

1936-39; 1940-41**.

1936-39; 1940-41**.

1936-39; $1940-41 * *$.

1936-39; $1940-42 * *$.

1912-17.

* Gage heights or gage heights and discharge measurements only.

* Only monthly figures of discharge or contents.

m/ In reports of State engineer. 
Rio Grande tributeries-Continued

Rio Chame (Chama River) at Park View, N. Hex....................... El Vado Reservoir near Tierra Amarilila, N. Hex...................... Rio Chama (Chama River at El Vado) (near Tierra Amarilla) below

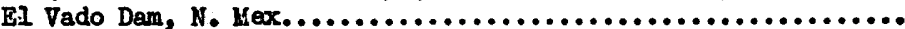

Rio Chama (Chama River) at Abiquiu, N. lex.........................

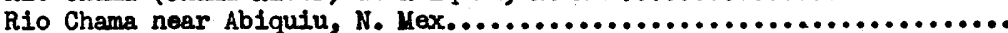

Rio Chama (Chama River) near (at) Chamita, N. Hex...................

Brazos River near Brazos, N. Hex............................

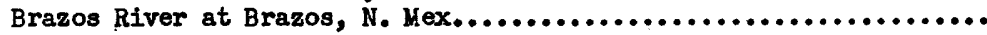

Iittle Brazos River near Brazos, N. Hex........................

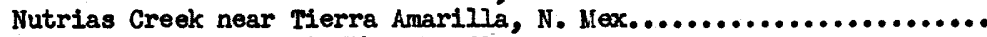

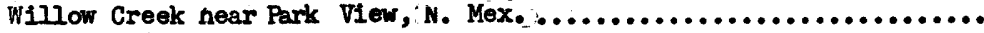

Nutrias Creek near Cebolla, N. Llex............................

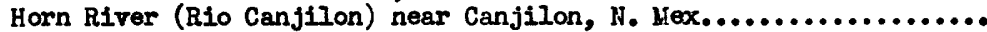

Bl Rito Creek near El Rito, N. Hex............................

Rio Ojo Caliente at Ia Madera, N. Hex...........................

Rio Vallecitos at Vallecitos, N. Hex............................

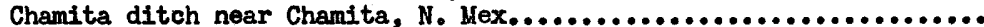

Santa Clara ditch near Espanola, N. Mex.........................

Rio Medio (head of Rio Santa Cruz) above Cundiyo, N. Mex..............

Rio Santa Cruz (Rio, Medio) at Cundiyo, N. Mex......................

Rio Santa Cruz (Rio Medio) above Chimayo, N. Hex.....................

Rio Santa Cruz (Rio Medio) at Chimayo, H. Hex......................

Rio Santa Cruz at Riverside, N. liex............................

Rio Frijoles at Cundiyo, N. Yex.............................

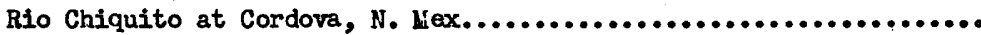

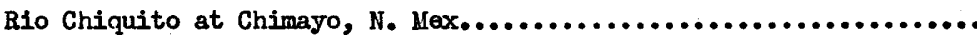

Santa Clara Creek near Espanola, N. Hex........................

Hill Acequia near Espanola, N. Mex............................

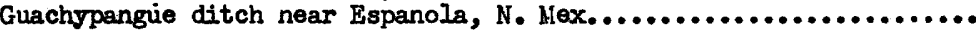

San Ildefonso ditch near Espanola, $N$. Hex.........................

Nambe Creek (head of Pojoaque Creek) near Nambe, N. Hlex................

Nambe Creek at Pojoaque Bridge near Nambe, N. Hex.....................

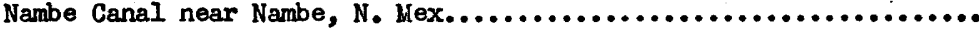

Ilano Frio ditch near Nambe, $N$. hiex............................

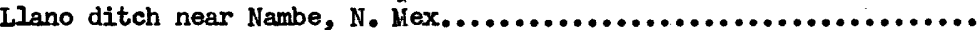

Mocha ditch near Nambe, No Mex.................................

Comunidad ditch near Nambe, N. Mex............................

Ortiz ditch at Nambe, N. Mex................................

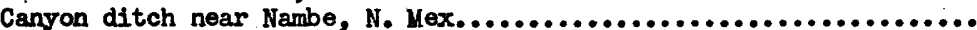

Acequia Rincon near Nambe, N. Nex..............................

Las Joyas ditch near Nambe, N. Mex.............................

Trujillo ditch near Nambe, N. Hex..............................

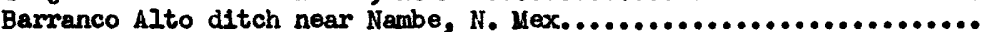

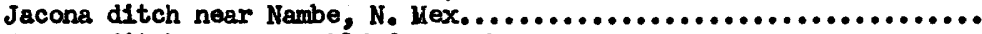

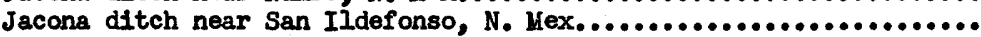

Rio Tesuque above diversions near Santa $\mathrm{Fe}, \mathrm{N}$. Mex.................

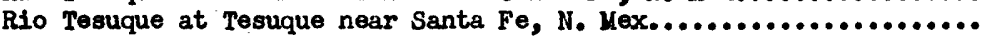

Rio Tesuque at Tesuque Bridge near Sants $\mathrm{Fe}, \mathrm{N}$. Kex.................

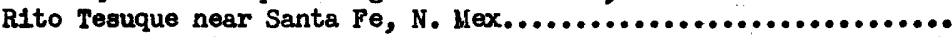

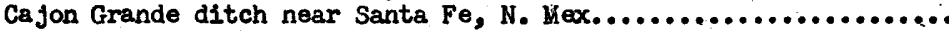

De La Cruz ditch near Santa $\mathrm{Fe}$, N. Hex.........................

Acequia Madre near Santa $\mathrm{Fe}$, N. Mex.............................

Acequia Medio near Santa $\mathrm{Fe}$, N. Mex.........................

Hubbard ditch near Santa Fe, N. llex..........................
Orainage Area

square miles)

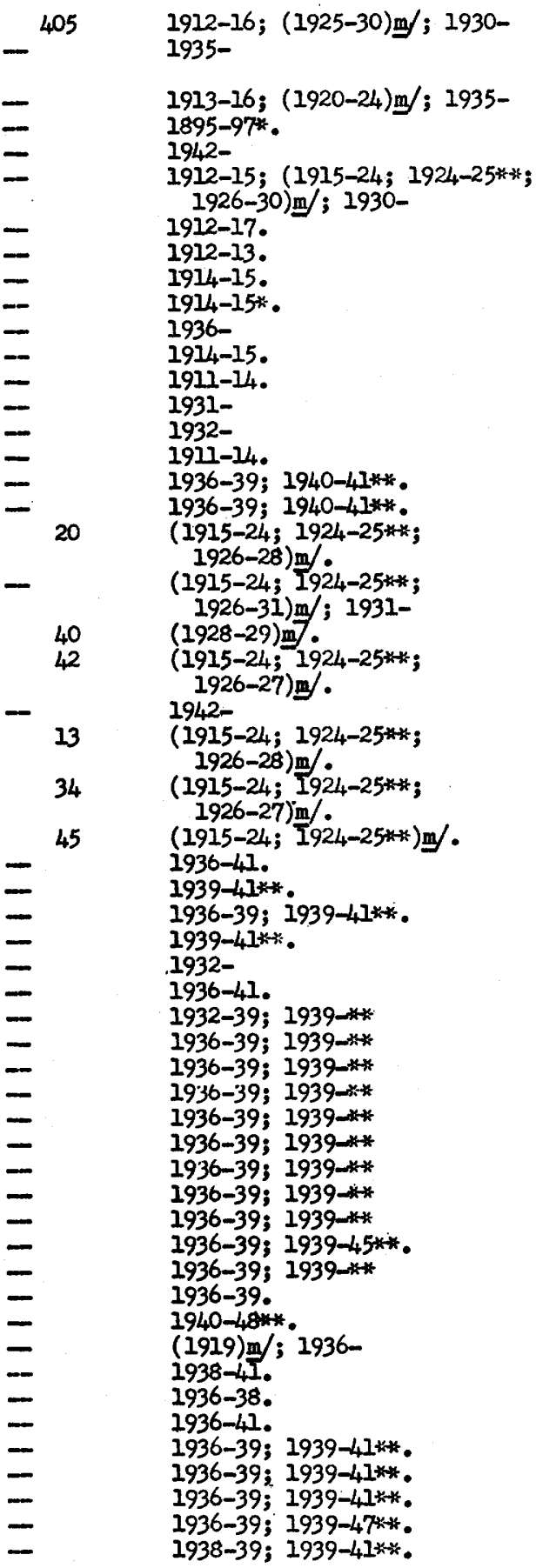

* Gage heights or gage heights and discharge measurecients only.

* only monthly figures of discharge or contents.

$\mathrm{m} /$ In reports of state engineer.

Note.- Many stations on canals and drafns in the Rio Grande Basin between Santa Fe and San Marcial, N. Mex., are not listed in this index. Records for those stations from 3936 to 1938 are published in Hater-Supply Papers 858 and 878 . 
io Grande tributaries-Continued

Nambe Creek tributariesm-Continued

Rio Tesuque tributaries--Continued

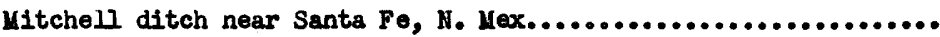

Post ditch near Tesuque Pueblo, N. Hex.........................

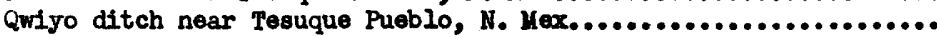

Corral ditch near Tesuque Pueblo, N, Hex........................

Del Barranco ditch near San Ildefonso, H. Mex......................

De La Otra Banda ditch near San Ildefonso, N. Yex.................

Rancho ditch near $\operatorname{San} I$ Ildefonso, $N$. Yex..........................

De Los Indios ditch near San Ildefonso, $N$, Mex....................

Well ditch near San Ildefonso, $N$. Kex.........................

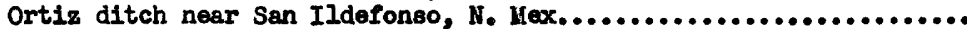

Sante $\mathrm{Fe}$ Creek at Monument Rock near Sante Fe, N. Mex................. McClure (Granite Point) Reservoir near Santa Fe, N. Mex................ Santa Fe Creek (above reservoly) near Santa Fe, N. Mex.................

Santa Fe Creek (below Pirst diversion) near Santa Fe, N. Yex.......... Nichols Reservoir near Sante Fe, N. Mex............................ Santa Fe Creek at Santa $\mathrm{Fe}, \mathrm{N}$. Mex.............................. Santa $\mathrm{Fe}$ Water and Iight $\mathrm{Co}$. ditch near Santa $\mathrm{Fe}, \mathrm{N}$. Mex............ Arroyo Hondo near Santa $\mathrm{Fe}, \mathrm{N}$. Mex.............................

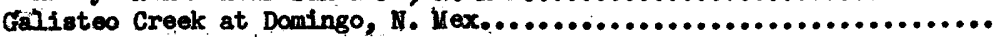
San Felipe east side acequia near Domingo, N. Mex................... Jemez Creek near Jemez, N. Mex.................................. Jemez Creek at San Yo1dro, N. Mex................................ Jemez Creek (Rio Jemez) near San Ysidro, H. Mex.....................

Jemez (Creek) River near Bernalillo, N. Hex....................... Rio Las Vacas near Cuba, N. Mex................................. Rio Guadalupe near Jemez Springs, N. Mex.......................... Rio Cebolls near Jemez Springs, N. Mex.......................... Jemez west side ditch near Jemez, N. Yex.......................... Jemez east side ditch near Jemez, N. Kex........................... Antonio Pecos ditch near Jemez, N. Mex............................ San Ysidro ditch near San Ysidro, N. Kex........................ Zia ditch near San Ysidro, N. Hex............................. Tijeras Creek near Albuquerque, N. Hex............................ Rio Puerco near Cabezon, N. Mex..................................

Rio Puerco at Cabezon, N. Yex.....................................

Rio Puerco near Guadalupe, N. Mex..................................

Rio Puerco at Rio Puerco, N. Kex.....................................

Rio Puerco near Bernardo (Ia Joya), N. Kex.......................... La Jara Creek near La Jara, N. Kex............................. Chico Arroyo near Guadalupe, N. Mex.............................. Bluewater Creek (head of San Jose River) near Bluewater, N. Hex.....

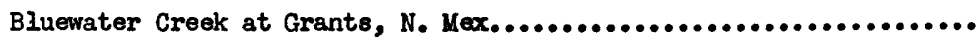

San Jose River near Grants, N. Mex................................ San Jose River near San Pidal, N. Mex........................... San Jose River near Casa Blanca, N. Mex........................... San Jose River near Laguna, N. Hex............................... San Jose River at Correo, N. Mex.............................. San Jose River near Sawanee, N. Mex.............................

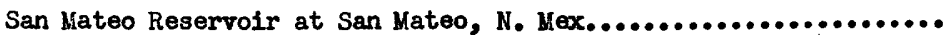
Encinal Creek near Casa Blanca, $N$. Mex.........................

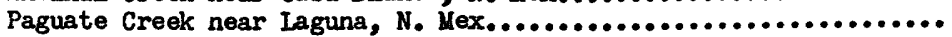
McCartys South SIde ditch near San Fidel, N. Mex................. McCartys North Side ditch near San Fidel, N. Hex..................
Drainage Area

(square miles)

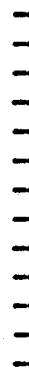

$=$

40

$-13.5$

$-$

$\overline{-}$

$-$

$\overline{\overline{ }}=$

1,860

5,160

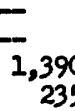

\section{Period of Record}

1936-39; 1939-**

1936-39; 1939-41**.

1936-39; 1939-41**.

1936-39; 1939-41**.

1936-39; 1939-4工**.

1936-39; 1939-41**.

1936-39; 1939-41**.

2936-39; 1939-41**.

1938-39; 1939-***

1936-39; 1939-41**.

1910.

$1947-$

1913-14; (1915-24; 1924-25**;

1926-30) $\mathrm{m} /$; 1930 -

1910; 1913*.

1942-

1907-10; 1912*。

1910*.

1913-14; (1s15-22)m/.

$1941-$

1936-39; 1939-41**.

1936-41.

1936-41.

(1923-24; 1924-25**;

$1926-27 *) \mathrm{m} /$.

1936-38; $1943-$

$1938-41$.

1938-41.

1939.

1936-39; 1939-4I**.

1936-39; 1939-41**.

1936-39; 1939-42**.

1936-39; 1939-4工*t*.

1936-39; 1939-41**.

(1921-22)m/; $1943-1949$.

1943-

1944-

1943.

1910-12*; 1913-14;

(1915-24; 1924-25*;

1926-27) $\mathrm{m} /$; 1934-

1911-14*; 1939-

1932-33.

1943-

1912-14; (1915-19; 1921-24;

1924-25**; 1926-30)m/; 1930 -

1912-13; 1914*; (1915-24;

1924-25**; 1926)m/; 19491936-

1936-41; 1950-

1936-41.

$1937-41$.

1943-

550 1910; 1911*; 1912-14;

$(1915-17) \mathrm{m} /$.

$1939-43 * k$.

1936-39; $1939-40 * *$.

1937-41.

1940-41**; 1950-**

1940-41**; 1950-***

* Gage heights or gage heights and discharge measurements only.

** Only monthly figures of discharge or contents.

$\mathrm{m} /$ In reports of state engineer.

Note.- Manj stations on canals and drains in the Rio Grande Basin between Santa Fe and San Marcial, N. Mex., are not listed in this index. Records for those stations from 1936 to 1938 are published in llater-Supply Papers 858 and 878 . 
Rio Grande tributarles-Continued

Rio Puerco tribateries--Contimued San/Jose River tributaries--Continued

Acomita Reservoir outlet near San Fidel, N. Hex................... Seama-Para je ditch near Casa Blanca, N. Mex...................... Casa Blance ditch at Casa Blanca, $N$. Hex........................ New Laguna ditch near New Laguna, $N$. Kex........................ Laguna ditch at New Laguns, N. Mex.......................... Mesita ditch near Leguna, $\mathbb{N}$. Yex............................. Paguate Reservolr outlet near Iaguna, N. Mex...................... New Iaguna ditch wasteway near Casa Blanca, N. Yex................ Rio Salado near San Acacia, N. Yex............................ Socorro main canal north at San Acacia, N. Mex...................... Socorro main canal south (at end) near San Antonio, N. Mex............ San Antonio Riveraide drain near San Antonio, N. Yex................ San Antonio Riverside drain near San Marcial, N. Hex................. Elmendorf Interior drain near San Antonio, $N$. Kex.................. Alamose River near Yonticello, $\mathbf{K}$. Yex........................ Las Palcmas River near Las Palcasa, N. Lex......................... Diversions trom the Rio Grende into The American Canal at

El Paso, Tex.........................................

Diversions from the Rio Grande Into Acquia Madre (Mexdcan

Canal) near Juarez, Chih., Yex.............................

Diversions from the Rlo Grande in the El Paso Valley, Tex........... Tornillo drain at mouth at Alamo Alto, Tex....................... Tornillo Canal at wasteway near Alamo Alto, Tex...................

Hudspeth Canal at head near Alomo Alto, Tex.....................

Rio Conchos at Cuchillo Parado, Chih., Yex........................

Rio Conchos near Ojinaga, Chih., Mex............................ of 22,600

Diveraions from Rio Grande between upper and Iower Preaidio

atation. Tex........................................

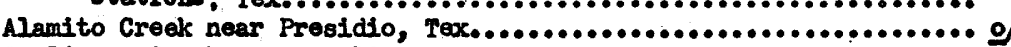

Terlinqua Creek near Terlinqua, Tex.......................... of

Lozier Creek near Langtry, Tex................................. of

Pecos River near Conles, N. Hex...............................

Pecos River (at Irvin's ranch near Cowles) near Pecos, N. Mex........

Pecos River neer San Jose, N. Hex...............................

Pecos RIver near Anton Chico, N. Mex............................

Pecos River at Santa Rosa, N. Mex.

Pecos River near Puerto de Luna, N. Yex.......................... Alamogordo Reservolr near (Gusdalupe) Fort Sunner, N. Mex........... Pecos River (near Guadalupe) below Alamogordo Dam, N. Hex...........

Pecos River near Fort Sumner, N. Mex............................

Pecos River near Acme, N. Yex................................ Pecos River neer Roswell, N. Yex............................... Pecos RIver near Iake Arthur, N. Kex............................ Pecos River near (Dayton) Artesia, N. Mex..........................

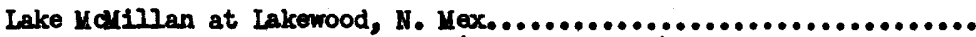
Pecos River below Mcllillan Dam (near Lakewood), N. Mex..............

Pecos River below Major Johnson Springs near Carlsbad, N. Mex....... Pecos River at dam site 3 near Carlsbad, N. Yex....................

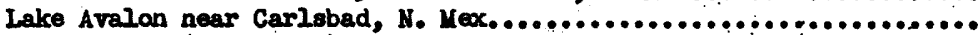
Pecos River (at Avalon) below Avalon .Dem, near Carlsbad, N. Mex.... Pecos River at Carlsbad, $\mathrm{H}$. Yex.................................

Pecos River near Malaga, N. Hex................................. Pecos River at Plerco Canyon crossing near Ualaga, N. Mex...........
2,650

3,970

4,390

4,390

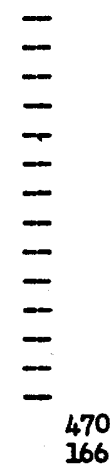

1,504

1,070 1,806 189

539
1,050

$-$

11,380

14,760

15,300

16,990

16,990

17,620

18,070

18,070

18,100

19,190 19,260
Period of Record

1938-40; 1940-41**.

1937-39; 1939-41**.

1937-39; $1939-41 * *$.

1937-39; 1939-41**.

1937-39; 1939-41**.

1936-39; 1939-41**.

1940-41**.

1937-39; $1939-41 * *$.

1948-

1936-41; 1941 - * *

1937-38; 1948-

1936-38; 1948-***

$1948-x *$

1936-38**; 1948-**

(1929) $\mathrm{y} / 1931-42$.

(1931) $\mathrm{y}$.

(1938-)o/

(2938-) $)$

$(1938-47) \mathrm{o} /$

1931-38.

(1930-31)m/; 1931-38.

(1930-31) $\mathrm{m} /$; 1931-38.

(1945-)o

(1924-) 0 /**

(1932-33)o/; 1934**o/.

(1932-) 0 )

(1932-)

(1932-35)o/.

1910-14; (1915-19)m/.

(1919-24; 1924-25*स;

$1926-30) \mathrm{m} /$; 1930

$1939-40$.

1910; 1911*; 1912-14;

(1915-24; 1924-25*;

1926-30) $\mathrm{w} /$; 1930 -

1903-05*; 1906; 1910-14;

(1915-24; 1924-25**;

1926-30)피 1930

1938-

1939 **

1912-14; (1915-24; . 1924-25**; 1926-30) $\mathrm{y}$; 1930

1904-10; 1912-13; (192123**) $\mathrm{m} /$.

$(1921-23 * *) \mathrm{m} / 1937$ -

1903-05*; 1906.

1938-

1905-25; (1925**; 1926-31) $\mathrm{g}$; 1931-

$1907 * ;$ 1939-**

1906-09; 1910-11*; 1939-40;

1946-

1947-

1939-40; $1944-$

1939-*

1906-0.7; 1940.

$1903-06 ; 1907-08 * ; 1914-25$;

$(1925-28) \mathrm{m} / ; 1928-30$;

$(1930-31) \mathrm{m} /$; $1931-$

$(1920-25) ;(1925-31) \mathrm{m} /$; $1931-$ 1938-41.

* Gage heights or gage heights and discharge messurements only.

* Only monthly figures of discharge or contents.

w In reports of state engineor.

of In International Boundary and Water Comission Water Bulletins.

Note.- Figures of drainage area shown for stations in the Pecos, River Basin represent the contributing area. 
$\therefore$ io Grande tributaries-Continued

Pecos River at Red Bluff, N. Mex...............................

Pecos River near Angeles, Tex...................................

Red Bluff Reservoir near orla, Tex...............................

Pecos River near 0rla, Tex.....................................

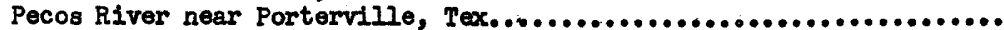

Pecos River above Barstow (above Barstor Canal), Tex..................

Pecos River above Barstow (below Barstow Canal), Tex.................

Pecos River near Pecos, Tex......................................

Pecos River at Pecos, Tex......................................

Pecos River near Barstow, Tex..................................

Pecos River below Barstow, Tex................................

Pecos River near Grandfalls, Tex..............................

Pecos River below Grandfalls, Tex.............................

Pecos River near Buena Vista, Tex...............................

Pecos River near Girvin, Tex..................................

Pecos River near Sheffield, Tex..................................

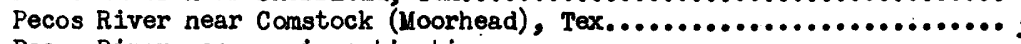

Pecos River seepage investigation...............................

Gallinas River near Montezuma, N. Mex..........................

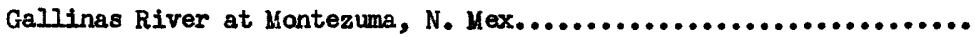

South Fork Gallinas River near El Porvenir, N. Bex............... Rio Ruidoso (head of Rio Hondo) near Ruidoso, N. Mex............... Rio Ruidoso near Glencoe, N. Mex............................... Rio Ruidoso at Hondo, N. Mex................................

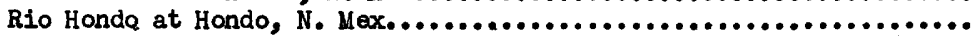
Rio Hondo at Diamond $A$ Ranch near Roswell, N. Hex................. Rio Hondo at Hondo Reservoir, N. Mex........................... Rio Hondo at Roswell, N. Mex................................

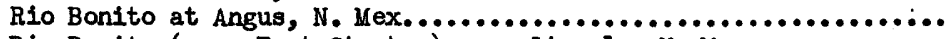

Rio Bonito (near Fort Stanton) near Lincoln, N. Mex..............

Rio Bonito at Hondo, N. Mex...............................

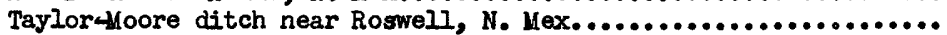

Hondo Reservoir inlet near Roswell, N. Ilex....................

Hondo Reservoir scour gate near Roswell, N. Hex................. Rio Felix at old highway bridge, near Hagerman, N. Mex............. Rio Felix near Hagerman, N. Mex.............................. Cottonwood Creek near Lake Arthur, N. Mex........................

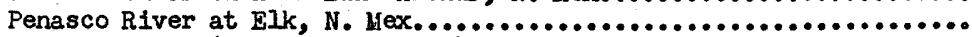
Penasco River (at Cleve's Ranch) near Elk, N. Hex................. Penasco River (at $Y .0$. Crossing) near Hope, N. Mex.................

Penasco River near Dayton, N. Mex............................ Black River above Malaga, N. Mex............................ Black River (near) at Malaga, N. Hex......................... Delaware River near Malaga, N. Mex............................. Delaware River near Red Bluff, N. Mex........................

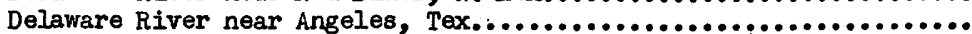
Seepage below east side Ked Bluff Dam near Orla, Tex............... Seepage below west side Red Bluff Dam near orla, Tex............... Salt (Screwbean) Draw near orla, Tex.......................... John Camp pump near Orla, Tex............................... Joe B. Neel pump near Riverton, Tex...........................

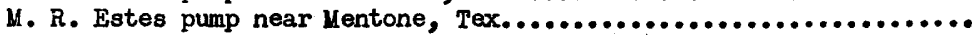
Reeves County Water Improvement District No. 2 Canal near

Mentone (Farmers Independent Canal near Porterville), Tex....... Ward County Water Improvement District No. 3 Canal near

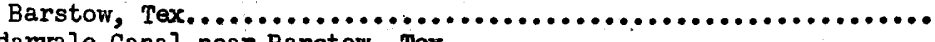

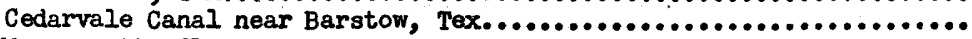
Margueretta flume near Pecos, Tex..............................

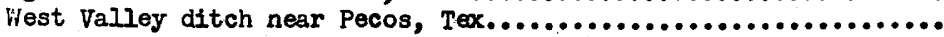

Vrainage Area

square miles)

\section{9,540}

20,540

20,720

21,300

$=$

$\bar{z}$

$=$

22,100

25,980

27,820

29,560

31,660

35,293

84

87

-

$-960$

$\bar{z}$

306

$-$

${ }^{1,92}$

1300

1,300

343

360

$-$

967

-

455

$\bar{z}$

\section{Period of Record}

1937-

1914-37.

1937-**

1937-

1922-26.

1916-21.

1922-26.

1898-1907.

1939-

$1914-15$.

1939-40.

1915-26.

1939-

1921-26.

1939-

1921-25; 1939-49.

1898*; 1900-31; (1931-)of

1918.

(1915-24; 1924-25**;

1926-30) m/; 1930-

1903*; 1904-14; (1915-24;

$1924-25 * * ; 1926-30) \mathrm{m} /$;

1930-

1911-14; (1915-20)m/.

1912*.

1910-il*.

1930-

1930-31.

(1908-09) m/; 1939

1903-05*; 1906 .

1903-05*; 1906.

(2908-09**) m/; 1930-31.

(1908-09**) $)$; $1930-31$.

1930-

1905.

$1906-09 \%$.

1906.

1939-

1932-39.

1932-

1910-11*.

1911*.

(1921-24; 1924-25**;

1926-29) $\mathrm{m} /$.

1905-06; 1906-08* .

1940; 1946 -

$1914-15$; $1939-40$.

1912-13.

1937-

$1914-15$.

1939-40.

1939-40.

1939-40; 1943-

1940 .

1940 .

1940.

1922-25; 1939-

1939-

1922-25.

1898 ; $1900-07$.

1904 .

* Gage heights or gage heights and discharge measurements only.

$* *$ Only monthly figures of discharge or contents.

m/ In reports of State engineer.

of In International Boundary and Water Commission Water Bulletins.

Note.- Figures of drainage area shown for stations in the Pecos River Basin represent the contributing area. 
Tio Grande tributaries-Continued

Pecos River tributaries-Continued

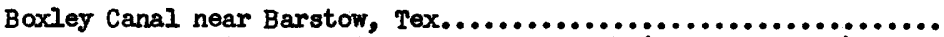

Ward County Irrigation District No. I Canal (Barstow Canal),

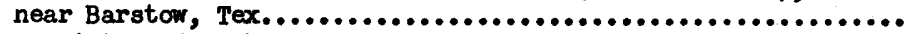

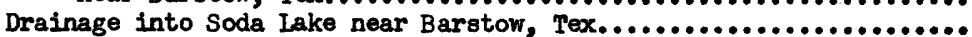

Reeves County Water Improvement District No. 2 Canal wasteway near Pecos, Tex............................................

John T. Yarborough pump near Pecos, Tex.......................

Ward County Irrigation District No. I lateral No. I wasteway near Pecos, Tex........................................

Barstow drainage ditch No. I near Barstow, Tex...................

Rock Quarry Draw near Barstow, Tex............................

Barstow drainage ditch below confluence of ditches 2,3 , and

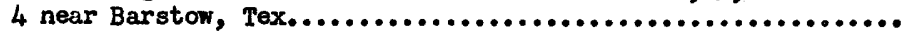

Ward County Irrigation District No. 1 Canal wasteway below

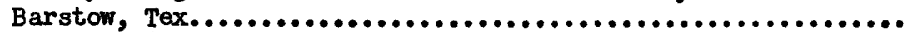

Madera Canyon (head of Toyah Creek) near Toyahvale, Tex............. Madera Canyon seepage investigation.......................... Toyah Creek near Pocos, Tex.................................. Toyah Creek below Toyah Lake near Pecos, Tex....................... Toyah Creek seepage investigation..............................

Little Aguja Canyon seepege investigation.....................

Big Aguja Canyon seepage investigation.......................

Phantom Lake Spring near Toyahvale, Tex........................

Giffin Springs at Toyahvale, Tex..............................

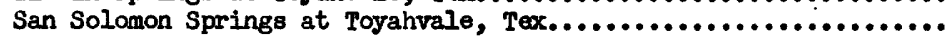

Hain Canal of Reeves County Water Improvement District No. 1 seepage investigation................................

Lateral Canals of Reeves County Water Improvement District No. 1 seepage investigation............................

Seepage investigations in Reeves County Water Improvement District No. 1, Tex.................................

Seepage investigations on laterals in Toyah Creek Basin, Reeves County, Tex....................................

Reservoir Outlet Canal of Reeves County Water Improvement District No. I seepage investigation......................

West Sandia Spring at Balmorhea, Tex..........................

West Sandia Creek seepage investigation......................

East Sandia Spring at Balmorhea, Tex.........................

Gherry Canyon seepage Investigation.........................

Salt Draw near Pecos, Tex................................

Iimpla Creek (head of Barrilla Creek) near Fort Davis, Tex........

Limpla Creek seepage investigation............................

Barrilla Creek near Saragosa, Tex..............................

Barrilla Creek near Pecos, Tex.............................. Grandfalls-Big Valley Canal near Barstow, Tex.................... E. W. Fate pump near Grandfalls, Tex........................... Pecos County Water Improvement District No. 2 upper diversion Canal (Imperial Highline Canal), near Grandfalls, Tex........

Pecos County Water Improvement District No. 2 Canal near

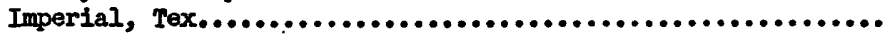

Ward County Water Improviement District No. 2 Canal near Grandfalle, Tex....................................

Pecos County Water Improvement District No. 2 lower diversion Canal (Imperial Lowline Canal), near Grandfalls, Tex..........

Pecos County Water Improvement District No. 3 Canal near Grandfalls, Tex......................................

Pecos County Water Improvenent District No. 3 Canal near Imperial, Tex......................................

Pecos County Water Improvement District No. 3 Canal below

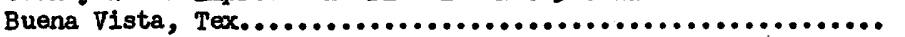

Ward County Water Improvement District No. 2 Iateral No. 2 wasteway below Grandfalls, Tex............................

Ward County Water Improvement District No. 2 Canal wasteway below Grandfalls, Tex.
Urainage Area

square miles)

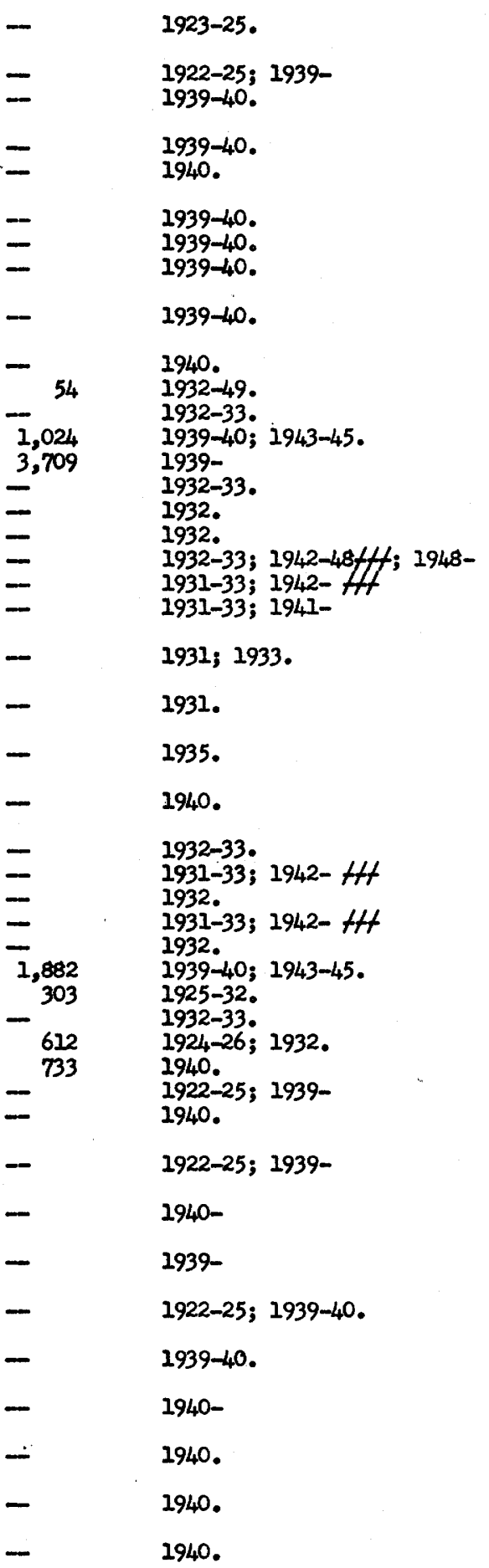

Af Discharge measurements only at regular intervals.

Note.- Figures of drainage area shown for stations in the Pecos River Basin represent the contributing area. 
dio Grande tributaries-Contimued

Pecos River tribataries--Continued

Drainage Area

(square miles)

Pecos County Water Improvement District No. 3 Canal wasteway

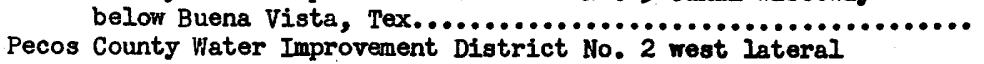
wasteway near Buena Vista, Tex..........................

Pecos County Vlater Improvement District No. 2 east lateral wasteway below Buena Vista, Tex...........................

Drainage from Powell Lake below Buens Vista, Tex.....................

Comanche Springs at Fort Stockton, Tex..........................

Leon Springs and flowing wella, 8 miles west of Fort Stockton, Tex.......................................

San Pedro Springs 7 miles northeast of Fort Stockton, Tex............ Seepage investigations on canals in Comanche Creek basin, Pecos County, Tex....................................

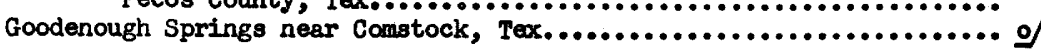

Devils River near Juno, Tex.......................................

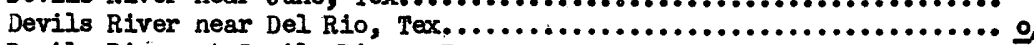

Devils River at Devils River, Tex................................

Devils River seepage investigation.............................

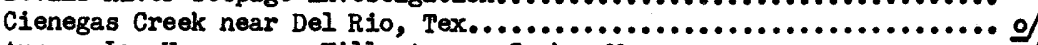

Arroya Las Vacas near Villa Acuna, Coah., Hex.....................

San Felipe Creek near Del Rio, Tex................................

Sycamore Creek near Del Rio, Tex.................................

Pinto Creek near Del Rio, Tex....................................

R10 San Diego at Jimenez, Coah., Mex...........................

Las Moras Creek near Eagle Pass, Tex...........................

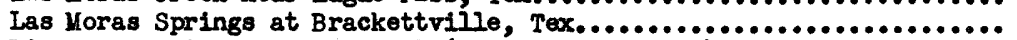

Rio San Rodrigo near El Moral (Paso De Las Nulas), Coah... Meo.......... Diversions from the Rio Grande into Maverick Canal extension

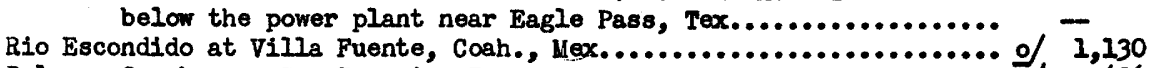

Dolores Creek near San Ignac10, Tex............................ of 1,1306

Rio Salado near Guerrero, Tamaulipas, Mex....................... of 21,528

El Tigre Arroyo near Zapata, Tex............................... of 261

Rio Alamo at Cd. Mier "El Paso del Contaro", Tam., Mex............... of 1,675

R1o San Juan near Santa Rosalia Ranch, Tam., Hex................... o 13,000

Rio San Juan at Ia Quemado, Tam., Mex..............................

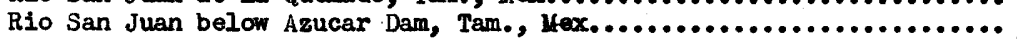

Rio San Juan above Rio Grande City, Tex...........................

Los Olmos Creek near Rio Grande City, Tex.........................

Rio San Juan below Rio Grande City, Tex.............................

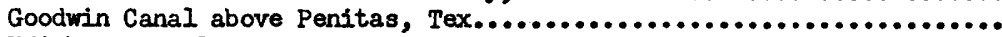

Edinburg Canal at Penitas, Tex.....................................

Kission Canal near Mission, Tex..................................

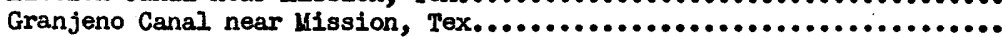

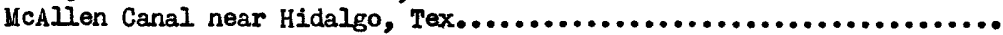

Rio Bravo Canal near Hidalgo, Tex.............................

South floodway south of McAllen, Tex...........................

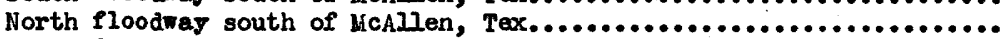

North floodwas near Sebastian, Tex............................. Diversions from Rio Grande into the Retamal Canal near Rio

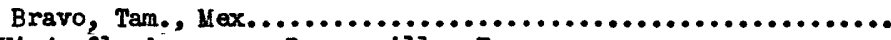

Rancho Viejo floodway near Brownsvilie, Tex......................

Diversions from Rio Grande on United States side below Rio

Grande City, Tex.

INTERIOR BASINS IN NEW IEXXICO

\section{MIMBRES BIVTR BASII}

Mimbres River near Mimbres, N. Mex.

(1921-24; 1924-25**; 1926-30)点/; 19301-

\footnotetext{
* Gage heights or gage heights and discharge measurements only.

** Only monthly figures of discharge or contents.

Af Discharge measurements only at regular intervals.

m/ In reports of State engineer.

of In International Boundary and Water Commission Water Bulletins.
}

Note.- Figures of drainage area shown for stations in the Pecos River bas in represent the contributing area. 
Drainage Area (square miles)

Limbres River near Faywood, N. Yex..............................

Bear Canyon near Uimbres, N. Hex............................... Lampbright Draw near Santa Rita, N. Mex........................... Whitewater (Draw) Creek at Hurley, N. Mex........................... Cameron Creek at Fort Bayard, N. Yex............................... Cameron Creek near Hurley, N. Mex.................................. Stevens Creek near Fort Bayard, N. Mex............................

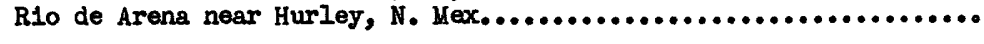

\section{TULAROSA VALLIT BASIX}

R10 Tularosa at Mescalero, N. Mex...............................

R10 Tularosa near Bent, N. Mex.................................

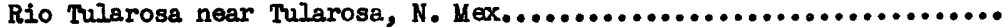

Rio Ia Luz on Coglan's Ranch, near Ia Iuz, N. Mex...................

Rio Ia Luz near La Luz, N. Yex..................................

Rio La Luz at La Luz, N. Mex.............................. A lamogordo-La Luz ditch at Ia Luz, N. Mex......................

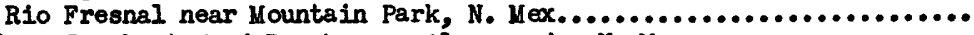

Alamo Creek at Wood Ranch near Alamogordo, N. Hex................... Alamogordo Water Supply (at intake) near Alamogordo, N. Mex.........
485

Period of Record

1908-10; 1912*; 1912-14;

(1915-24; 1924-25**;

2926-30)m/; 1930

1937-

1912-14; $(1915-22) \mathrm{m}$.

$1913-14 *$.

1907-10; 1911-13*。

1913-11*.

$1907-14 *$.

1913-14*.

1910-11*.

1911*; 1948 -

1912-14; (1916-17)m/; 1931-

(1912**)m

30 1911-12*.

- 1910-13*; 1931-32。

1934-41; 1941-49**.

44 1911-12*; (1912**)m/。

- 1931-37.

1932-41; 1941-k*

* Gage heights or gage heights and discharge measurements only.

** Only monthly figures of discharge or contents.

m/ In reports of State engineer. 


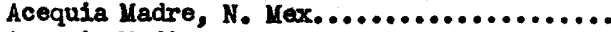

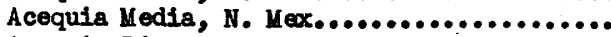
Acequia Rincon, N. Mex...................... Acomita Reservolr outlet, N. Mex........... Addicks Reservolr, Tex.................... Alamito Creek, Tex.......................

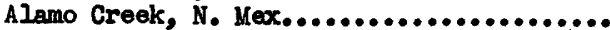

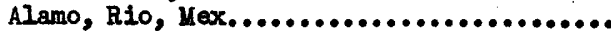
Alamogordo-Ia Lus ditch, N. Mex............

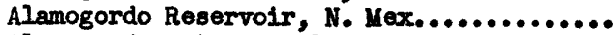
Alamogordo water supply, N. Kex............ Alamosa Creek, Colo........................

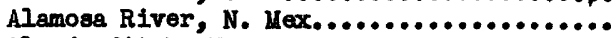

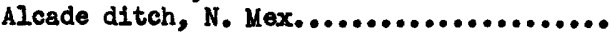
Allre ditch, Colo........................ American Canal Co.'s Canal, Tex............. Angeline River, Tex.................... Antonio Pecos ditch, N. Mex................. Aquilla Creek, Tex.......................

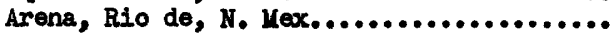
Arena Creek, Colo.......................... Arenoso Creek, Tex....................... Arroyo Hondo, N, Mex...................... Arroyo Las Vacas, Mex...................... Atascosa River, Tex..................... Attoyac Bayou, Tex........................ Austin Bayou, Tex......................... Aytsh Bayou, Tex........................

Barker Reservoir, Tex.................... Barranco Alto ditch, N. Mex................. Barrilla Creek, Tex..................... Barstow Canal, Tex. See Ward County

Irrigation D1strict No. I Canal.

Barstow drainage ditches, Tex.............. Barton Creek, Tex......................... Barton Springs, Tex...................... Bayou Anacoco, La.......................... Bayou Des Connes, La........................ Bayou Nezplque, La....................... Bayou Plaquemine Brule, La................. Bayou San Miguel, I8..................... Bear Canyon, N. Yex....................... Bockwith Creek, La........................ Big Aguja Canjon seepage inveatigation,

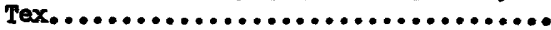

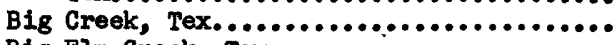

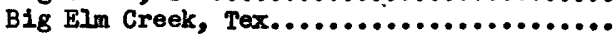
Big Sandy Creek (tributary to Sabine River), Tex......................... Big Sandy Creek (tributary to Trinity River), Tex.........................

Big Spring Creek, Colo................... Black River, N. Mex........................ Blanco River, Tex.......................... Bluewater Creek, N. Mex.................... Bluff Creek, Tex........................... Bonfto, Rio, N. Mex...................... Bosque River, North, Tex.................. Bosque River, South, Tex................. Bowen drain, Colo........................ Bowles Creek seepage investigation, Tex.... Boxley Canal, Tex......................... Brady Creek, Tex........................ Brays Bayou, Tex........................ Brazos River, N. Mex..................... Brazos River, Tex....................... Brazos River, Clear Fork, Tex.............. Brazos River, Double Mountain Fork, Tex... Brazos River, Little, N. Mex................

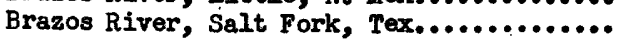

20,21

Brazos Valley Irrigation Co.'s canal. See American Canal Co.'s canal.

Bridgeport Reservoir, Tex.......................

Brushy Creek, Tex...........................

Brown County Water Improvement District

No. I Canal, Tex........................... Browmood Reservoir, Tex........................ Buchanan Reservoir, Tex....................... Bull Creek, Tex............................. Buffalo Bayou, Tex............................. Buridick Creek, La............................

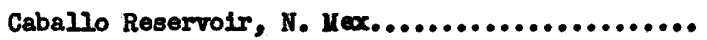
Cabresto Creek, N. Mex........................... Cajon Grande ditch, N. Yex..................... Calcasien RIver, La............................ Cameron Creek, N. Yex........................ Caney Creek, Tex.............................. Canyon ditch, $\mathrm{N}$. Mex........................... Carnero Creek, Colo............................

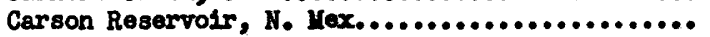
Casa Blanca ditch, N. Kex...................... Castas Creek, N. Hex............................ Cebolla, Rio, H. Kex........................ Cedar Creek, Tex..............................

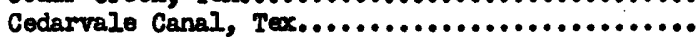
Cerro Canel, Colo.-N, Yex....................... Chama, Rio, N. Irex.......................... Chama River, H. Mex.......................... Chambers Creek, Tex............................ Chamita ditch, N. Mex......................... Champlin Creek, Tex.......................... Cherokee Bayou, Tex.......................... Cherry Canyon seepage investigation, Tex......... Chico Arroyo, N. Mex............................

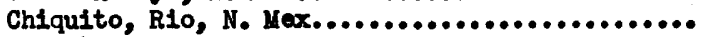
Chocolate Bayou, Tex......................... Cibolo Creek, Tex............................. Clenegas Creak, Tex........................... Clear Creek, Colo.............................

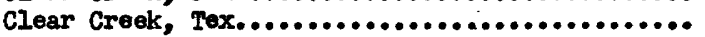
Clear Fork Brazos River, Tex..................... Clear Fork Trinity River, Tex.................. Coleto Creek, Tex............................. Colorado, Rio, N. Mex...........................

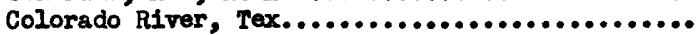

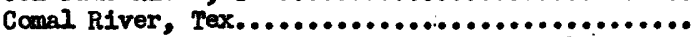
Comanche Creek Basin, seepage from canals, Tex.. Comanche Springs, Tex......................... Comunidad ditch, N. Mex..................... Concho River, Tex..........................

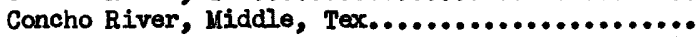
Concho River, North, Tex........................ Concho River, South, Tex........................ Conchos, Rio, Mex,............................ Conejos River, Colo............................ Cordillera ditch, Colo......................... Corrall ditch, N. Mex........................

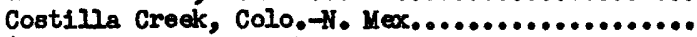

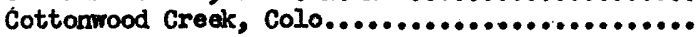
Cottonwood Creak, N. Yex...................... Corhouse Creek, Tex..........................

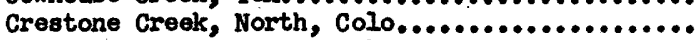
Crestone Creek, South, Colo......................

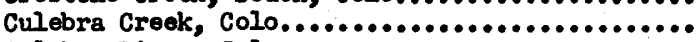
Culebra River, Colo............................. Cypress Creek, La.-Tex........................

Deadman Creek, Colo............................. Deep Creek, Tex............................... Deer Creek, Tex............................. 
De Ia Cruz ditch, N. Yex.................. De La Otra Branda ditch, N. Mex............

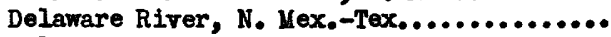
Del Barranco ditch, N. Kex.................. De Los Indos ditch, N. Mex................. Denton Creek, Tex......................... Devils River, Tex.......................... Diversions from R10 Grande, Tex..........13,15,16 Dolores Creek, Tex..................... 16 Double Mountain Fork Brazos River, Tex..... Dove Creek, Tex.......................... Drainage into Soda Lake, Tex............... Dry Creek, Tex........................... Dry Frlo River, Tex.......................

Eagle Mountain Reservoir, Tex.............. Eastdale No: 1 intake, Colo................. East Fork or Branch. See name of main stream.

East Sandia Spring, Tex.................. Edinburg Canal, Tex........................ Elephant Butte Reservoir, N. Mex............ Elm Creek, Tex........................... Elm Creek, Big, North, Tex...............

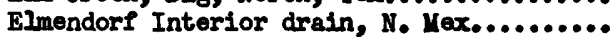
Elm Fork Trinity River, Tex...............

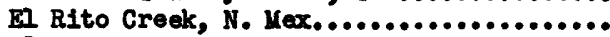

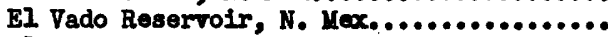
El TIgre Arroyo, Tex..................... Embudo Creek, $\mathrm{N}$. Hex..................... Empire Canal, Colo..................... Encinal Creek, N. Mex......................

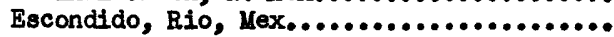
Estes, M. R., pump, Tex....................

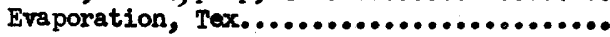

Fairchild Creek, Tex..................... Farmers Independent Canal, Tex............. Farmers Union drain, Colo................. Fate, E. W., pump, Tex...................

Felix, Rio, N. Hex...................... Fernando de Taos, Rio, N. Yex................ Fort Phantom Hili Reservoir, Tex.......... Fresnal, Rio, N. Mex.....................

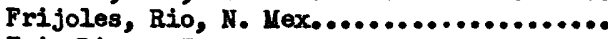
Frio River, Tex.......................... Frio Iake Outlot, Tex....................

Galisteo Creek, N. Yex.....................

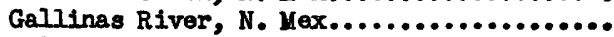
Gallinas River, South Pork, N. Yex......... Giffin Springs, Tex.................... Goodenough Springe, Tex.................. Goodwin Canal, Tex...................... Goose Creek, Colo........................ Grande, Rio, Colo.-N. Yex.-Tex.............

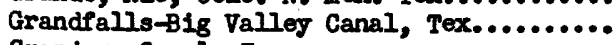
Granjeno Canal, Tex....................... Guachupangue ditch, N. Mex.................

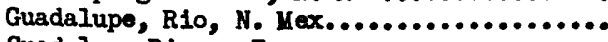
Guadalupe River, Tex.....................

Hickory Branch, La...................... Hickory Slough, Tex.....................

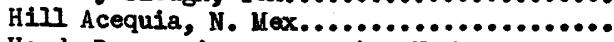
Hondo Reservoir scour gate, N. Mex......... Hondo Reservoir inlet, N. Hex.............. Hondo, Rio (tributary to Pecos River),
11

12.

14

12

12

3

16
Hondo, Rio (tributary to Rio Grand9),

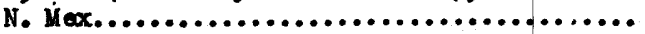

Hords Creek, Tex.............................

Horn River, $N$. Mex...........................

Hubbard ditch, N. Mex........................

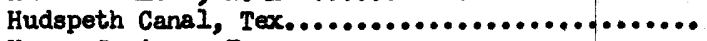

Hueco Springs, Tex..........................

Imperial Highline Canal, Tex.................. Indian ditch, N. Yex......................... Isle du Bols Creok, Tox........................

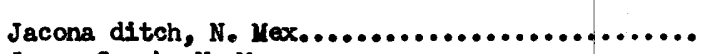

Jemez Creek, N. Mex. ..........................

Jemez east side ditch, N. Mex.....................

Jemez west side ditch, $N$. Hex....................

John Camp pump, Tex........................

Johnson Creek, Tex...............................

Johnson Fork Llano River, Tex....................

Juan Yanuel ditch, N. Kex......................

Kerber Creek, Colo..........................

La Garits Creek, Colo............................

Laguna ditch, N. Mex.........................

La Jara Creek, Colo..........................

La Jara Creek, N. Mex..........................

La Jara drain, Colo..........................

Lake Avalon, N. Mex..........................

Lake Cherokee, Tex. ...........................

Lake Colorado City, Tex.......................

Lake Corpus Christi, Tex......................

Lake Dallas, Tex..........................

Lake Fork Sabine River, Tex...................

Lake Mcuilian, N, Mex........................

Lake Nasworthy, Tex...........................

Lake Tyler, Tex............................

Ia Luz, Rió, N. Mex..........................

Lampasas River, Tex.........................

Lampbright Draw, N. Mex......................

Las Joyas ditch, N. Mex........................

Las Moras Creak, Tex...........................

Las Moras Springs, Tex........................

Las Palamas River, N. Kex......................

Las Vacas, Rio, No Mex.........................

Lateral Canals of Reeves County Water

Improvement District No. 1, Tex..............

Latir Creek, N. Mex.........................

Iavaca River, Tex..........................

Leon River, Tex...........................

Leon Springs, Tex.........................

Leona River, Tex..............................

Iimpia Creek, Tex.............................

Ittlo Aguja Canyon seepage investigation, Tex..

Iittle Brazos River, N. Yex....................

Ifttle River, Tex........................

Ilttle Spring Creek, Colo....................

Ifttle Walnut Creek, Tex.......................

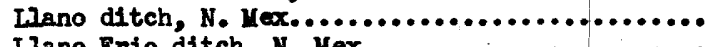

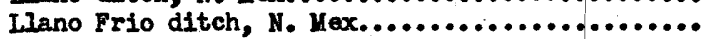

ILlano River, Tex...........................

Llano River, North, Tex.......................

Lozter Creek, Tex...........................

Los 0 lmos Creek, Tex..........................

Los Pinos River, Colo..........................

Lucero, Rio, N. Mex.........................

Madera Canyon, Tex...........................

Main Canal of Reeves County Water Improvement District No. I, Tex... 


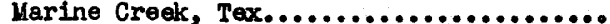
Margueretta flume, Tex.................... Marshall Ford Reservoir, Tex............. LcCartys north side ditch, N. Mex......... McCartys south side ditch, N. Yex.......... McClure Reservoir, N. Mex................

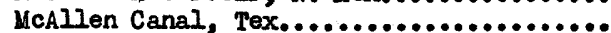
Modillan, Lake, N. Mex....................

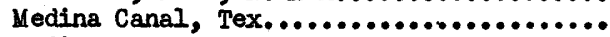
Medina Reservoir, Tex.................... Medina River, Tex...................... Medio, Rio, N. Mex........................ Mese ditch, Colo....................... Mesita ditch, N. Mex..................... Uiddle Concho River, Tex................. Middle ditch, Colo...................... Mimbres River, N. Mex................... Mission Canal, Tex......................

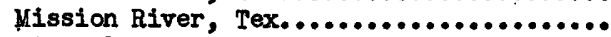

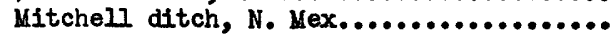

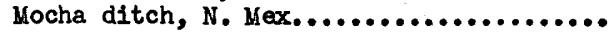
Morgan Creek, Tex...................... Mountain Creek, Tex.................... Ifud Creek, Tex........................

Nambe Canal, N. Mex..................... Nambe Creek, N. Mex...................... Navasota River, Tex.................... Navidad River, Tex...................... Neches River, Tex....................... Neel, Joe B., pump, Tex.................. New Laguna ditch, N. Hex................. New Laguna ditch wasterray, N. Mex.......... New Mexdco Branch-Cerro Canal, Colo....... Nichols Reservoir, N. Hex.................. Nolands River, Tex..................... North Bosque River, Tex................... North Concho River, Tex................. North Crestone Creek, Colo................. North Elm Creek, Tex................... North floodwas south of HcAlien, Tex..... North floodray near Sebastian, Tex....... North lateral Farmers Union ditch, Colo............................ North Llano River, Tex.................... North Sapato Creek, Colo.................. Noyes Canal, Tex........................ Nueces River, Tex....................... Nueces River, West, Tex................... Nutrias Creek, N. Mex....................

Oak Creek, Tex......................... Ojo Caliente, Rio, N. Mex.................. Onion Creek, Tex...................... Ortiz ditch, N. Mex....................... Oyster Creek, Tex.......................

Paguate Creek, N. Hex..................... Paguate Reservoir outlet, N. Yex.......... Paint Creek, Tex.......................

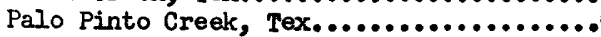
Paluxy Creek, Tex........................ Peach Creek (tributary to East Fork San Jacinto River), Tex............. Peach Creak (tributary to Guadalupe

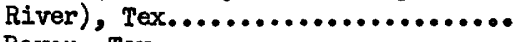
Pecan Bayou, Tex....................... Pecos County Water Improvement District No. 2 canals.....................
Pecos County Water Improvement District

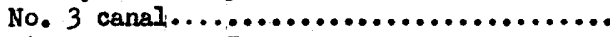

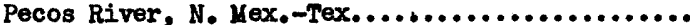
Pedernales River, Tex........................

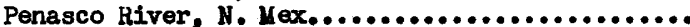
Phantcm Lake Spring, Tex......................

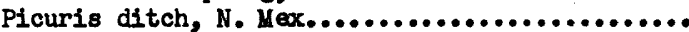
Pinos Creek, Colo...........................

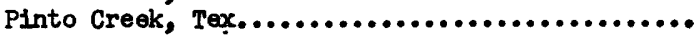
Plum Creek, Tex............................

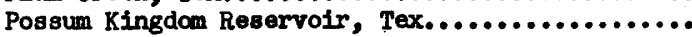
Post ditch, N. Mex......................... Powell Lake, Tex., drainage from.................

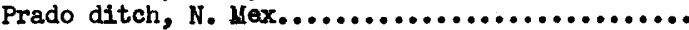
Prairie ditch, Colo........................... Pueblo Creek, N. Mex............................ Pueblo de Taos, Rio, N. Mex.................... Puerco, Rio, N. Mex.........................

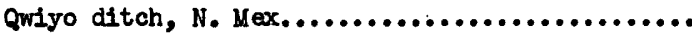

15,16

13,14

Rancho ditch, N. Mex........................ Ranchos, Rio, de Taos, N. Mex...................

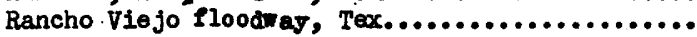

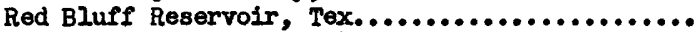
Red River (Rio Colorado), N. Mex................. Reeves County Water Improvement District

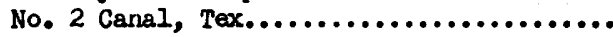
Reeves County Water Improvement District No. 2 Canal wasteway, Tex............... Reeves County Water Improvement District No. I seepage investigation, Tex.......... Reservoir Outlet Canal of Reeves County Water Improvement District No. 1, Tex............

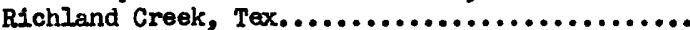
Richmond Irrigation Co.'s Canal, Tex.............

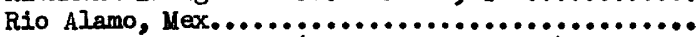
Rio Alamosa, Colo. (See Alamosa Creek).......... Rio Bonito, N. Hex............................

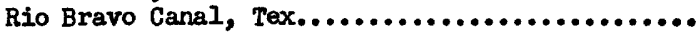
Rio Cebolla, N. Mex..........................

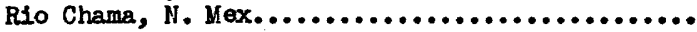
Rio Chiquito, N. Yex.........................

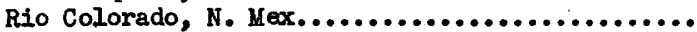
Rio Conchos, Mex............................

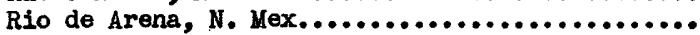
Rio Escondido, N. Mex........................

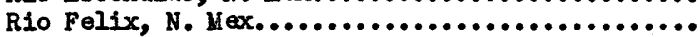

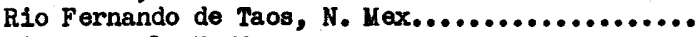

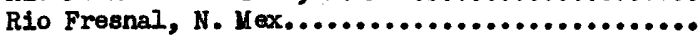
Rio Frijoles, N. Mex.......................

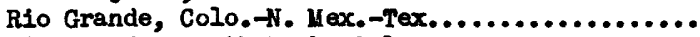

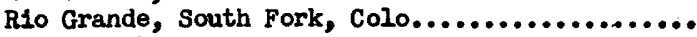
Rio Guadalupe, N. Mex........................ Rio Hondo (tributary to Pecos River), N. Mex.... Rio Hondo (tributary to Rio Grande), N. Hex.....

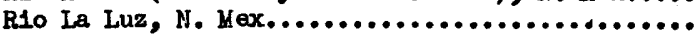
Rio Las Vacas, N. Hex......................... R10 Lucero, N. Kex.............................

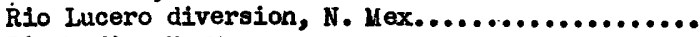

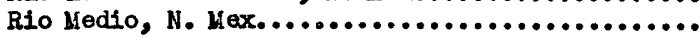
Rio Ojo Caliente, N. Nex.....................

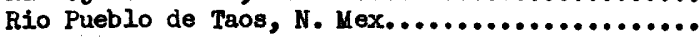
Rio Fueblo de Taos, north channel, N. Mex........ Rio Puerco, N. Jex.........................

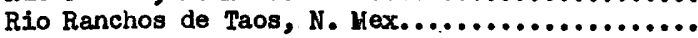

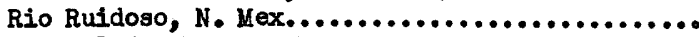

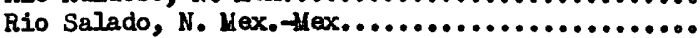
Rio San Antonio, Colo. See San Antonio River... 
Rio San Diego, Hex..................... Rio San Juan, Mex..................... Rio San Rodrigo, Mex..................... Rio Santa Cruz, N. Hex.................. Rio Taos, N. Mex...................... Rio Tesuque, N. Mex.................... Rio Tularosa, N. Hex................... Rio Vallecito, N. Mex.................. Rito Tesuque, N. Mex.................... Rock Creek, Colo...................... Rock Quarry Draw, Tex................... Ruidoso, Rio, N. Mex....................

Sabinal River, Tex.................... Sabine River, La. - Tex.................. Sabine River, Lake Fork, Tex............. Sagusche Creek, Colo....................

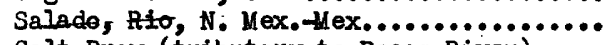
Salt Draw (tributary to Pecos River), Tex........................... Salt Draw (tributary to Toyah Creek), Tex............................. Salt Fork Brazos River, Tex............. San Antonio River, Tex.-Colo.............. San Antonio Riverside drain, N. Mex....... Sand Creek, Colo. See Arena Creek........ Sandia Spring, East, Tex................

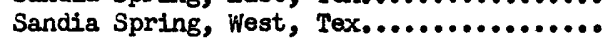

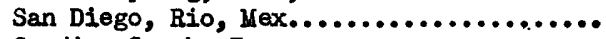
Sandies Creek, Tex...................... Sandy Creak, Big (tributary to Sabine River), Tex....................... Sandy Creek, Big (tributary to Trinity River), Tex.................

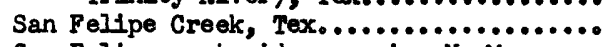
San Felipe east side acequia, N. Mex...... San Francisco Creek, Colo................. San Gabriel River, Tex.................. Sangre de Cristo Creek, Colo............... San Ildefonso ditch, N. Hex.............. San Jacinto River, Tex................... San Jacinto River, East Fork, Tex......... San Jacinto River, West Fork, Tex........ San Jose River, N. Mex................... San Juan, R1o, Mexdco................... San Luis Creek, Colo..................... San Marcos River, Tex.................. Sạn Marcos Springs, Tex.................. San Mateo Reservoir, N. Mex............... San Pedro Creek, Tex.................... San Pedro Springs (Fort Stockton), Tex... San Rafael ditch, N. Mex.................. San Rodrigio, Rio, Mex.................

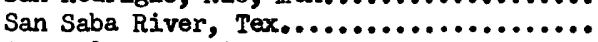
San Solomon Springs, Tex.................

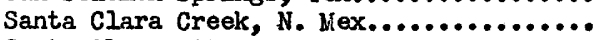
Santa Clara ditch, N. Hiex................ Santa Cruz, Rio, N. Mex.................. Santa Fe Creek, N. Mex.................. Santa Fe water \& Light Co. ditch, N. Kex. Santistevan Creek, N. Hex............... San Ysidro ditch, N. Mex................. Screwbean Draw, Tex. See Salt Draw...... Seama-Paraje ditch, $\mathrm{N}$. Viex................ Seco ditch, N. Nex..................... Seepage below Red Bluff Dam, Tex......... Seepage investigation on laterals in

Toyah Creek basin, Tex..............

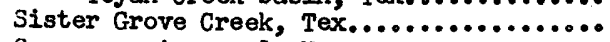
Socorro main canal, N. Hex............... Soda Lake, drainage into, Tex.............

\section{6}

16

16

11

10

11
South Bosque River, Tex....................... South Concho Irrigation Co.'s cenal, Tex......... South Concho River, Tex...................... South Crestone Creek, Colo..................... South floodray: south of McAllen, Tex............ South Fork or Branch. See name of main stream.

South LLano, Tex............................ Spanish Creek, Colo........................... Spring Creek (tributary to Middle Concho River), Tex............................... Spring Creek (tributary to San Jacinto River), Tex............................. Stevens Creek, N. Mex.......................

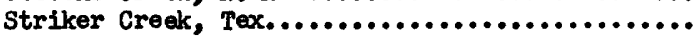
Sulphur Creek seepage investigations, Tex........ Sycamore Creek, Tex........................

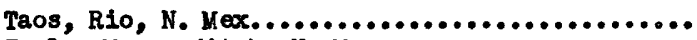
Taylor-Hoore ditch, N. Mex..................... Tenorio ditch, N. Mex........................ Terlingue Creek, Tex.........................

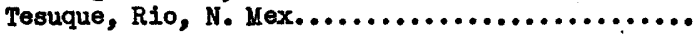

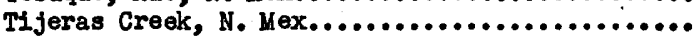

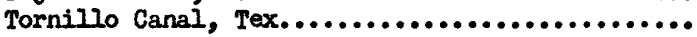

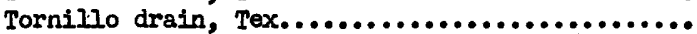
Toyah Creek, Tex............................

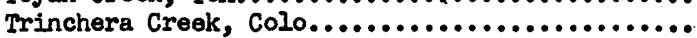
Trinity River, Tex.........................

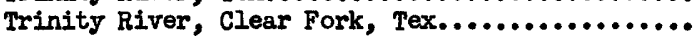
Trinity River, East Fork, Tex..................

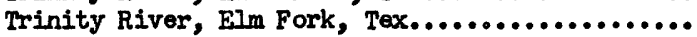

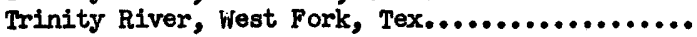

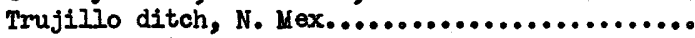

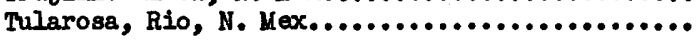

Ute Creek, Colo.............................. Ute Creek, N. Nex..........................

Vallecitos, Rio, N. Mex........................ Village Creek (tributary to Neches River), Tex... Village Creek (tributary to Trinity River), Tex..................................

Walnut Creek, Little, Tex..................... Ward County Irrigation District No. I Canal, Tex. Ward County Irrigation District No. I Canal

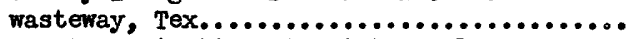
Ward County Irrigation District No. 1 lateral No. I wasteway, Tex................ Ward County Water Improvement District No. 2

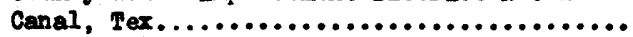
Ward County Water Improvement District No. 3

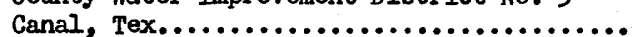

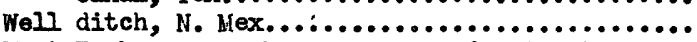
West Fork or Branch. See name of main stream... West Nueces River, Tex...................... Hest Sandia Creek seepage Investigation, Tex.... ilest Sandia Spring, Tex...................... ilest Valley ditch, Tex.....................

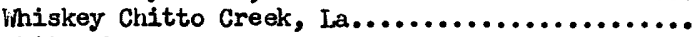
Whiteoak Bayou, Tex......................... White River, Tex..........................

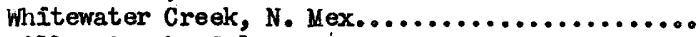

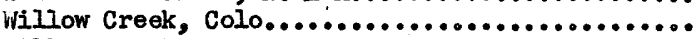

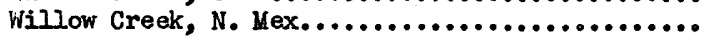

Yarborough, John T., pump, Tex................. Yegua Creek, -Tex......................... Zia ditch, N. Mex.......................... 
